\title{
Tetrahedron
}

journal homepage: www.elsevier.com/locate/tet

\section{Enantioselective rhodium-catalysed insertion of trifluorodiazoethanes into tin hydrides}

\author{
Stephen Hyde, ${ }^{a}$ Janis Veliks, ${ }^{a}$ David M. H. Ascough, ${ }^{a}$ Robert Szpera, ${ }^{a}$ Robert S. Paton, ${ }^{a, b}$ Véronique Gouverneur*a \\ ${ }^{[a]}$ Department of Chemistry, University of Oxford, Chemistry Research Laboratory, Mansfield Road, OX1 3TA, UK ${ }^{[b]}$ Department of Chemistry, Colorado State University, Fort Collins, CO 80523, USA.
}

\section{ARTICLE INFO}

Article history:

Received

Received in revised form

Accepted

Available online

\section{Keywords:}

Stannane

Asymmetric insertion

trifluorodiazoethane

Silane

Germanium hydride

\section{ABSTRACT}

Aryl substituted 2,2,2-trifluorodiazoethanes undergo rhodium(II)-catalysed insertion reactions with tin hydrides affording the corresponding $\alpha$-(trifluoromethyl)benzyl stannanes. This reactivity contrasts with that of diazo esters which predominantly afford $\mathrm{CH}_{2}$ reduction products in the presence of tin hydrides. The first example of asymmetric insertion into tin hydrides using diazo compounds is also described. In addition, this system extends to asymmetric germanium hydride and silane insertion.

\section{Introduction}

The insertion of diazo compounds into carbon-hydrogen and heteroatomhydrogen bonds is well-documented. ${ }^{1,2,3,4}$ Insertion reactions where $\mathrm{X}-\mathrm{H}$ ( $\mathrm{X}=$ $\mathrm{C}, \mathrm{Si}, \mathrm{S}, \mathrm{O}, \mathrm{N}, \mathrm{B}, \mathrm{P})$ bonds are cleaved by $\mathrm{Rh}, \mathrm{Cu}$, or other metal carbenoid intermediates, have been well studied both in racemic ${ }^{5,6,7,8,9,10,11}$ and asymmetric ${ }^{12,13}$ settings. Insertion into trialkyltin hydrides has received relatively little attention, despite several early reports supporting the feasibility of such insertion with carbenes. ${ }^{14,15,16}$ In 1995, Landais and coworkers reported an isolated example of trialkyltin hydride insertion with diazo esters (Figure 1a). ${ }^{17}$ Wang later showed that tin hydride enables the reduction of diazo carbonyl compounds, a process resulting from in situ protodestannylation of the tin hydride insertion product (Figure 1b). ${ }^{18}$. More recently, the groups of Wang and $\mathrm{Bi}$ have disclosed insertion into the $\mathrm{Sn}-\mathrm{H}$ bond leading to stable benzyltributylstannanes. ${ }^{19,20}$ Both methods generate the diazo intermediate in situ from a tosyl- or nosylhydrazone, however neither system offer a route to asymmetric insertion (Figure $1 \mathrm{c}$ and $1 \mathrm{~d}$ ). Our group extended $\mathrm{X}-\mathrm{H}$ insertion to trifluorodiazoalkanes. ${ }^{21}$ Under $\mathrm{Cu}$ catalysis, $\mathrm{Si}-\mathrm{H}, \mathrm{S}-\mathrm{H}, \mathrm{N}-\mathrm{H}, \mathrm{B}-\mathrm{H}$, and $\mathrm{P}-\mathrm{H}$ bonds were successfully cleaved, and an asymmetric protocol was developed for silanes and boranes (Figure 1e). However, tributyltin hydride gave a low yield of the insertion product under our standard reaction conditions. Herein, we report the successful development of a rhodium catalyzed tributyltin hydride insertion with arylsubstituted 2,2,2-trifluorodiazoethanes, as well as extension to an asymmetric catalytic protocol (Figure 1f). This system is also suitable for asymmetric insertion into germanium hydrides and silanes.

\section{Results and discussion}

The starting materials for this study, aryl-substituted 2,2,2 trifluorodiazoethanes, were prepared according to previously reported literature procedures. ${ }^{22,23}$ Optimization studies of the tributyltin hydride insertion reaction were performed using 4-(1-diazo-2,2,2-trifluoroethyl)-1,1'biphenyl (1a) as the model substrate (Table 1). Under our previously reported conditions, ${ }^{21}$ involving slow addition over one hour of diazo compound to a mixture of $\mathrm{Cu}(\mathrm{MeCN}){ }_{4} \mathrm{PF}_{6}$ (4 mol\%) and tributyltin hydride ( 2 equivs), only $26 \%$ of the desired product $2 \mathrm{a}$ was obtained, in addition to the reduced product.

a) Rh (II) catalysed Sn-H insertion with an unsubstituted $\alpha$-diazoester (1995, Landais)

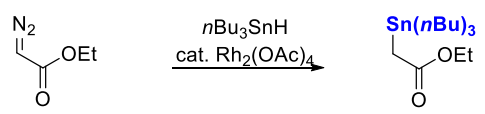

b) $\alpha$-Diazo cabonyl reduction with tributyltin hydride $(2000$, Wang)

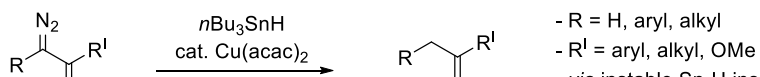

$$
\begin{aligned}
& \text { - via instable Sn-H insertion } \\
& \text { intermediates }
\end{aligned}
$$

c) Transition-metal-free stannylation with $\boldsymbol{N}$-tosylhydrazones (2017, Wang)

$$
\begin{aligned}
& n \mathrm{Bu}_{3} \mathrm{SnH}
\end{aligned}
$$

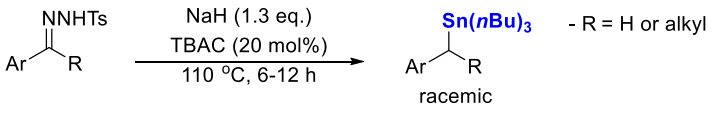

d) Silver (I) promoted insertion with $\mathbf{N}$-nosylhydrazones (2017, Bi)

$$
\begin{aligned}
& \mathrm{R}_{3}^{\mathrm{l}} \mathrm{X}-\mathrm{H} \quad-\mathrm{R}=\mathrm{H} \text {, alkyl, aryl }
\end{aligned}
$$

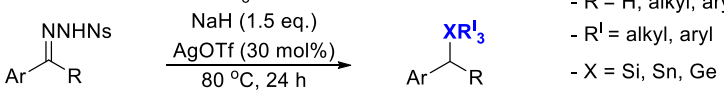

e) $\mathrm{Cu}$ (I) catalysed X-H insertion with trifluorodiazoalkanes (2016, Gouverneur)

$$
\begin{aligned}
& \mathrm{N}_{2} \quad \mathrm{R}^{\prime} \mathrm{X}-\mathrm{H} \quad \mathbf{X}^{\mathbf{2}} \quad-\mathrm{R}=\mathrm{H} \text {, aryl }
\end{aligned}
$$

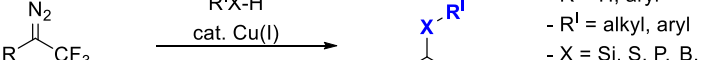

$$
\begin{aligned}
& { }_{\mathrm{CF}_{3}}-\mathrm{X}=\mathrm{Si}, \mathrm{S}, \mathrm{P}, \mathrm{B}, \mathrm{N} \\
& \text { up to } 98 \% \text { ee } \quad \text { - not suitable for } \mathrm{Sn}-\mathrm{H}
\end{aligned}
$$

f) This work: Rhodium catalysed insertion of trifluorodiazoethanes into $\mathrm{Sn}-\mathrm{H}$ bonds

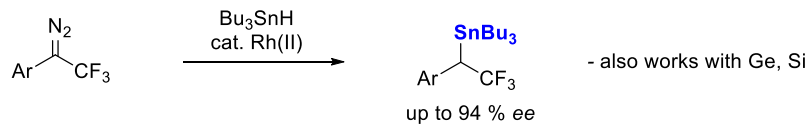

Figure 1. Sn-H insertion reactions with trialkyltin hydrides 
(Table 1, entry 1). Decreasing the amount of $n \mathrm{Bu}_{3} \mathrm{SnH}$ to 1.0 equivalents and changing the order of addition were not beneficial (Table 1, entries 2 and 3). Under $\mathrm{Rh}_{2}(\mathrm{OAc})_{4}$ catalysis, comparably poor results were obtained (entry 4). When the reaction was performed by fast addition of $\mathrm{Rh}$ (II) catalyst to an equimolar mixture of diazo compound $1 \mathrm{a}$ and $n \mathrm{Bu} \mathrm{u}_{3} \mathrm{SnH}$ in dichloromethane in an open vial, almost instantaneous evolution of nitrogen and disappearance of the characteristic orange colour of the diazo compound 1a was observed (Table 1, entry 5). The desired insertion product 2 a was isolated in $64 \%$ yield. $\mathrm{Cu}(\mathrm{II})$ and $\mathrm{Rh}(\mathrm{I})$ were ineffective for this transformation (entries 6 and 7). Screening various solvents highlighted dichloromethane as the optimum solvent for $\mathrm{Sn}-\mathrm{H}$ insertion (entry 5 vs. entries 8-11).

Table 1. Optimization studies for $\mathrm{Sn}-\mathrm{H}$ insertion ${ }^{[a]}$

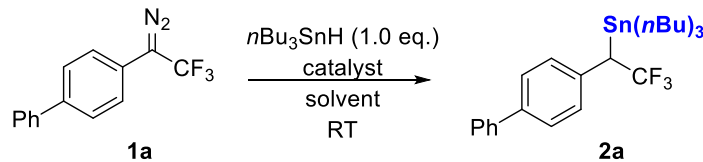

\begin{tabular}{|c|c|c|c|c|}
\hline Entry & Catalyst & Solvent & Procedure ${ }^{[c]}$ & Yield $^{[d]}$ \\
\hline $1^{[b]}$ & $\mathrm{Cu}(\mathrm{MeCN})_{4} \mathrm{PF}_{4}$ & $\mathrm{CH}_{2} \mathrm{Cl}_{2}$ & A & 26 \\
\hline 2 & $\mathrm{Cu}(\mathrm{MeCN})_{4} \mathrm{PF}_{4}$ & $\mathrm{CH}_{2} \mathrm{Cl}_{2}$ & B & 4 \\
\hline 3 & $\mathrm{Cu}(\mathrm{MeCN})_{4} \mathrm{PF}_{4}$ & $\mathrm{CH}_{2} \mathrm{Cl}_{2}$ & C & traces \\
\hline 4 & $\mathrm{Rh}_{2}(\mathrm{OAc})_{4}$ & $\mathrm{CH}_{2} \mathrm{Cl}_{2}$ & A & 25 \\
\hline 5 & $\mathrm{Rh}_{2}(\mathrm{OAc})_{4}$ & $\mathrm{CH}_{2} \mathrm{Cl}_{2}$ & C & 64 \\
\hline 6 & $\mathrm{Cu}(\mathrm{OAc})_{2}$ & $\mathrm{CH}_{2} \mathrm{Cl}_{2}$ & C & traces \\
\hline 7 & $\mathrm{Rh}(\mathrm{PPh})_{3} \mathrm{Cl}$ & $\mathrm{CH}_{2} \mathrm{Cl}_{2}$ & C & (6) \\
\hline 8 & $\mathrm{Rh}_{2}(\mathrm{OAc})_{4}$ & $\mathrm{CHCl}_{3}$ & C & (43) \\
\hline 9 & $\mathrm{Rh}_{2}(\mathrm{OAc})_{4}$ & Toluene & C & (59) \\
\hline 10 & $\mathrm{Rh}_{2}(\mathrm{OAc})_{4}$ & Hexane & C & (43) \\
\hline 11 & $\mathrm{Rh}_{2}(\mathrm{OAc})_{4}$ & DCE & C & (45) \\
\hline 12 & $\mathrm{Rh}_{2}(\mathrm{OAC})_{4}$ & $\mathrm{CH}_{2} \mathrm{Cl}_{2}$ & D & 74 \\
\hline 13 & $\mathrm{Rh}_{2}(\mathrm{OAc})_{4}^{[\mathrm{e}]}$ & $\mathrm{CH}_{2} \mathrm{Cl}_{2}$ & D & 67 \\
\hline 14 & $\mathrm{Rh}_{2}(\mathrm{OAc})_{4}^{[f]}$ & $\mathrm{CH}_{2} \mathrm{Cl}_{2}$ & D & 74 \\
\hline
\end{tabular}

[a] All reactions performed on a $0.1 \mathrm{mmol}$ scale with 1.0 eq. of $1 \mathrm{a}$ and $n \mathrm{Bu}_{3} \mathrm{SnH}$, with 4.0 mol\% catalyst loading, unless stated otherwise. [b] 2.0 eq. of $n \mathrm{Bu}_{3} \mathrm{SnH}$. [c] Procedure A: Syringe pump addition of $1 \mathrm{a}$ solution to a mixture of cat. and $n \mathrm{Bu}_{3} \mathrm{SnH}$ in dichloromethane over $1 \mathrm{~h}$. Procedure B: Syringe pump addition of $1 \mathrm{a}$ and $n \mathrm{Bu}_{3} \mathrm{SnH}$ solution to a catalyst suspension in dichloromethane over $1 \mathrm{~h}$. Procedure $\mathrm{C}: \mathbf{1 a}$ and $n \mathrm{Bu}_{3} \mathrm{SnH}$ added to vial and dissolved in dichloromethane (not degassed). The catalyst was then added. Procedure $\mathrm{D}: \mathbf{1 a}$ and $n \mathrm{Bu}_{3} \mathrm{SnH}$ added to a vial and dissolved in dichloromethane under anhydrous conditions. In a separate vial was added the catalyst and dichloromethane. The mixture of $1 \mathrm{a}$ and $n \mathrm{Bu}_{3} \mathrm{SnH}$ was added to the catalyst suspension. [d] All yields are those of isolated products unless stated otherwise. ${ }^{1} \mathrm{H}$ and ${ }^{19} \mathrm{~F}$ NMR yields, using ethyl trifluoroacetate as internal reference, are shown in parenthesis. [e] 2 mol\% of catalyst. [f] $10 \mathrm{~mol} \%$ of catalyst.

When the reaction was performed under an argon atmosphere with degassed dichloromethane, and the reagent mixture quickly transferred to the catalyst under inert atmosphere, the yield of the desired product $\mathbf{2 a}$ was further improved to $74 \%$ (entry 12 ). The use of 4 mol\% of $\mathrm{Rh}_{2}(\mathrm{OAc})_{4}$ was optimal for this transformation (entry 12 vs. 13 and 14).

After optimisation of the reaction conditions, the substrate scope was investigated. Various substituents such as alkyl, aryl, halide, and $\mathrm{CF}_{3}$ are tolerated on the phenyl group giving good to moderate yields of the insertion products 2. The limitations for this transformation are highlighted by the electron rich methoxy- substituted substrate $\mathbf{1} \mathbf{j}$ which gives a low yield of $38 \%$.
This procedure was also efficient for $\mathrm{PhMe}_{2} \mathrm{SiH}$ and $n \mathrm{Bu}_{3} \mathrm{GeH}$ insertion, delivering silane $\mathbf{2 m}$ and organogermanium $\mathbf{2 n}$ in good yields.

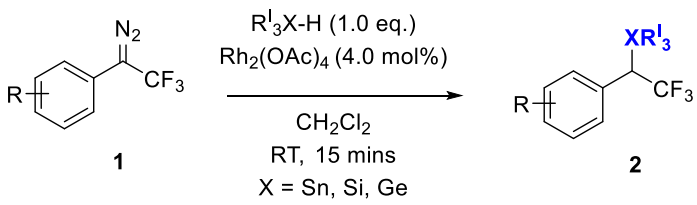<smiles>CCCCCC(c1ccc(C(=O)OCCCC)cc1)C(CCCCC)(c1ccc(Br)cc1)C(C)(C)C</smiles><smiles>CCCCCC(c1ccc(C)cc1)C(F)(F)F</smiles>

2d, $65 \%$<smiles>CCCCC(c1cccc(Br)c1)C(F)(F)F</smiles>

2e, $59 \%$<smiles>CCCCC(c1ccccc1)C(F)(F)F</smiles>

2f, $49 \%$<smiles>CCCCC(CCCC)C(c1ccc(F)cc1)C(F)(F)F</smiles>

$2 \mathrm{~g}, 54 \%$<smiles>CCCCC(CCCC)C(CCCC)(C(CCCC)c1ccc(C(F)(F)F)cc1)C(F)(F)F</smiles>

2h, $62 \%$<smiles>CCCCC(c1ccc(F)c(Cl)c1)C(F)(F)F</smiles>

$2 \mathrm{i}, 52 \%$<smiles>CCCCC(c1ccc(OC)cc1)C(F)(F)F</smiles>

$2 \mathrm{j}, 38 \%$<smiles>CCCCC(c1ccc(C)c(F)c1)C(F)(F)F</smiles>

$\mathbf{2 k}, 60 \%$<smiles>CCCCC(c1ccc(C(C)C)cc1)C(F)(F)F</smiles>

2I, $51 \%$<smiles>CC(C)[SiH2]C(c1ccc(-c2ccccc2)cc1)C([Al-]c1ccccc1)C(F)(F)F</smiles><smiles>CCCCC(c1ccc(-c2ccccc2)cc1)C(CC(C)(C)C)(C(F)(F)F)C(F)(F)Cl</smiles>

Scheme 1. Rhodium-catalysed insertion into Sn-H bonds with aryl substituted trifluorodiazoethanes. All reactions performed on $0.1 \mathrm{mmol}$ scale following procedure $D$ (table 1) unless stated otherwise. All yields are those of isolated products. [a] Procedure C. [b] 1.0 eq. of $\mathrm{PhMe}_{2} \mathrm{SiH}$ instead of $n \mathrm{Bu}_{3} \mathrm{SnH}$. [c] 1.0 eq. of $n \mathrm{Bu}_{3} \mathrm{GeH}$ instead of $n \mathrm{Bu}_{3} \mathrm{SnH}$.

When these reaction conditions were applied to diazo esters $\mathbf{3} \mathbf{a}$ and $\mathbf{3} \mathbf{b}$, the crude ${ }^{1} \mathrm{H}$ NMR spectra indicated mixtures of stannanes 4 and reduction products $\mathbf{5}$, and upon workup, only the latter were isolated (scheme 2 ). These examples highlight the instability of $\alpha$-carbonyl stannanes, which is consistent with previous literature. ${ }^{18}$

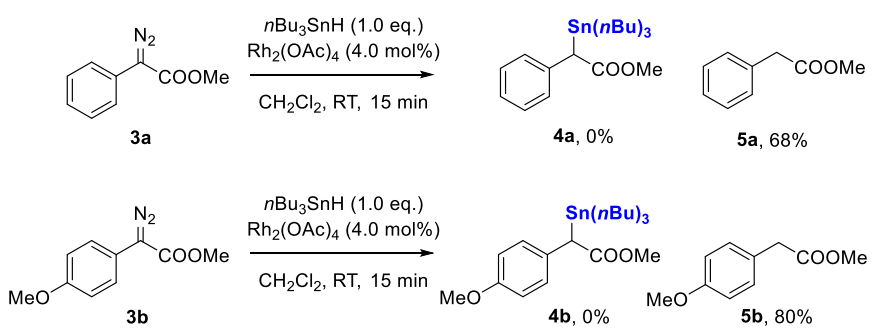

Scheme 2. Reactivity of diazo esters towards $n \mathrm{Bu}_{3} \mathrm{SnH}$

Given that Wang proposed that $\alpha$-carbonyl stannanes undergo protodestannylation via a putative radical pathway, radical stabilization enthalpies (RSE), which are derived from bond dissociation enthalpies (BDE), may account for the difference in stability of the stannane products. Due to the paucity of experimental tin bond dissociation enthalpies, we performed quantum mechanical calculations in Gaussian 09 rev. D.0.1. ${ }^{24}$ Computation of tin bond dissociation enthalpies is challenging, due to relativistic effects, and 
lack of experimental data for benchmarking. Grindley and Boyd have benchmarked organotin bond dissociation energies, finding a strong method dependence; we use the B3LYP/SDB-aug-cc-pVTZ//B3LYP/def2-SVPD level of theory described by the latter..$^{25,26}$ We also computed the corresponding proto derivatives using the higher G4 method for comparison and confirm good correlation of RSEs (see SI). ${ }^{27}$

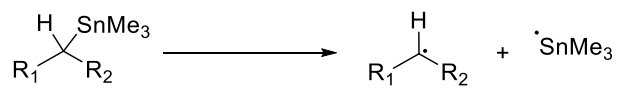

Table 2. Computed bond enthalpies in $\mathrm{kcal} / \mathrm{mol}^{[\mathrm{a}]}$

\begin{tabular}{llllll}
\hline Entry & Stannane & $\mathbf{R}_{\mathbf{1}}$ & $\mathbf{R}_{\mathbf{2}}$ & BDE & RSE \\
\hline 1 & $\mathbf{6 a}$ & $\mathrm{H}$ & $\mathrm{H}$ & 63.6 & 0.0 (defined) \\
2 & $\mathbf{6 b}$ & $\mathrm{H}$ & $\mathrm{CO}_{2} \mathrm{Et}$ & 58.6 & 5.0 \\
3 & $\mathbf{6 c}$ & $\mathrm{C}_{6} \mathrm{H}_{5}$ & $\mathrm{CF}_{3}$ & 47.2 & 16.4 \\
4 & $\mathbf{6 d}$ & $\mathrm{C}_{6} \mathrm{H}_{5}$ & $\mathrm{CH}_{3}$ & 41.8 & 21.8 \\
5 & $\mathbf{6 e}$ & $\mathrm{C}_{6} \mathrm{H}_{5}$ & $\mathrm{CO}_{2} \mathrm{Me}$ & 42.7 & 20.9
\end{tabular}

[a] Values computed at the B3LYP/SDB-aug-cc-pVTZ//B3LYP/def2-SVPD level of theory, BDE - bond dissociation enthalpy, RSE - radical stabilization energy.

Of the compounds calculated, the RSE corresponding to stannane $\mathbf{6 b}$ (5.0 $\mathrm{kcal} / \mathrm{mol}$ ) is lower than the other compounds investigated by at least 10 $\mathrm{kcal} / \mathrm{mol}$ (Table 2, entry 2). This is consistent with the low propensity for this compound to undergo protodestannylation via a radical pathway. In contrast, the phenyl-substituted ester $6 \mathrm{e}$ has a much higher RSE $(20.9 \mathrm{kcal} / \mathrm{mol})$ consistent with the protodestannylation observed experimentally (Table 2 , entry 5). ${ }^{18}$ Compound $6 \mathrm{c}$ has significantly lower RSE than the non-fluorinated derivative $6 \mathrm{~d}$ with RSEs of $16.4 \mathrm{kcal} / \mathrm{mol}$ and $21.8 \mathrm{kcal} / \mathrm{mol}$ respectively (Table, entries 3 and 4). This is consistent with reports of the destabilizing effect of trifluoromethyl substitution on carbon-centered radicals. ${ }^{28}$ Additionally, we find the RSE corresponding to $\mathbf{6} \mathbf{d}$ and the phenyl substituted ester $\mathbf{6 e}$ to be similar (RSE $=21.8 \mathrm{kcal} / \mathrm{mol}$ and $20.9 \mathrm{kcal} / \mathrm{mol}$ respectively).

Despite asymmetric heteroatom-hydrogen insertion reactions with diazo compounds having been extensively studied for many nucleophiles, ${ }^{12}$ to date there are no examples of catalytic enantioselective $\mathrm{Sn}-\mathrm{H}$ insertion reactions. This is possibly due to $\alpha$-diazo carbonyl compounds generating unstable stannane insertion products. ${ }^{18}$ Since aryl 2,2,2-trifluorodiazoethanes are converted into stable $\mathrm{Sn}-\mathrm{H}$ insertion products, we sought to develop an asymmetric protocol.

Optimization studies for an asymmetric insertion reaction were performed by testing a range of chiral $\mathrm{Rh}$ (II) catalysts (Table 3, entries 1 to 4). For operational simplicity, the chiral catalyst was added to a solution of diazo compound 1 a and tributyltin hydride in dichloromethane in an open vial, a process which allowed for the rapid identification of $\mathrm{Rh}_{2}$ (S-tertPTTL) 4 as the most efficient catalyst affording $\mathbf{2 a}$ in $90 \%$ ee but low yield (entry 4$).{ }^{29,30}$ When the reaction was performed under inert atmosphere in degassed solvent, a significant improvement of yield was achieved in addition to slight increase of enantioselectivity (entry 5). Decreasing temperature improved ee, however the yield was diminished (entries 6 and 7). At $-78{ }^{\circ} \mathrm{C}$, enantioenriched stannane $\mathbf{2 a}$ was isolated in $57 \%$ yield and $94 \%$ ee.
Table 3. Optimization studies for asymmetric insertion 1 a into $n \mathrm{Bu}_{3} \mathrm{SnH}^{[\mathrm{a}]}$

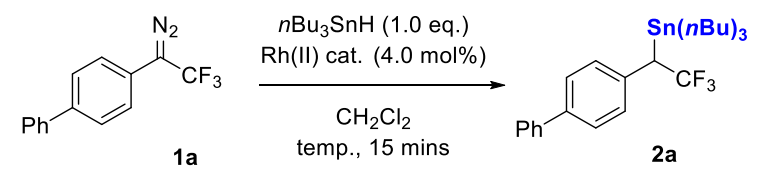

\begin{tabular}{llllll}
\hline Entry & Procedure & Catalyst & T/ ${ }^{\circ} \mathbf{C}$ & Yield 2a/\% & ee/ \% \\
\hline 1 & $\mathrm{~A}$ & $\mathrm{Rh}_{2}(\mathrm{~S}-\mathrm{DOSP})_{4}$ & $\mathrm{RT}$ & 78 & 7 \\
2 & $\mathrm{~A}$ & $\mathrm{Rh}_{2}(R-\mathrm{PTAD})_{4}$ & $\mathrm{RT}$ & 19 & 79 \\
3 & $\mathrm{~A}$ & $\mathrm{Rh}_{2}(R-\mathrm{BTPCP})_{4}$ & $\mathrm{RT}$ & 78 & -26 \\
4 & $\mathrm{~A}$ & $\mathrm{Rh}_{2}(\mathrm{~S} \text {-tertPTTL })_{4}$ & $\mathrm{RT}$ & 36 & -90 \\
5 & $\mathrm{~B}$ & $\mathrm{Rh}_{2}(\mathrm{~S} \text {-tertPTTL })_{4}$ & $\mathrm{RT}$ & 82 & -91 \\
6 & $\mathrm{~B}$ & $\mathrm{Rh}_{2}(\mathrm{~S} \text {-tertPTTL })_{4}$ & 0 & 74 & -92 \\
7 [c] & $\mathrm{B}$ & $\mathrm{Rh}_{2}(\text { S-tertPTTL })_{4}$ & -78 & 57 & -94 \\
\end{tabular}

Yields are those of isolated products. [a] Procedure A: Diazo and stannane added to vial and dissolved in dichloromethane. To the mixture was added the catalyst. Procedure B: (anhydrous conditions) Diazo and stannane added to a vial and dissolved in dichloromethane. In a separate vial was added the catalyst and more dichloromethane. The diazo/stannane mixture was added to the catalyst suspension. [c] Reaction time 30 minutes.

Comparable results were achieved when electron poor ester $\mathbf{1 c}$ and electron rich 4-methoxy substituted diazo compound $\mathbf{1 j}$ were subjected to the asymmetric insertion protocol, affording stannanes $\mathbf{2 c}$ and $\mathbf{2} \mathbf{j}$ with good to moderate yields with good enantioselectivities. In addition, these optimized reaction conditions were highly efficient for $\mathrm{PhMe}_{2} \mathrm{SiH}$ and $n \mathrm{Bu}_{3} \mathrm{GeH}$ asymmetric insertion, affording silane $\mathbf{2} \mathbf{m}$ and organogermanium $\mathbf{2 n}$ in good and excellent yields respectfully, both with $99 \%$ ee (scheme 3 ).
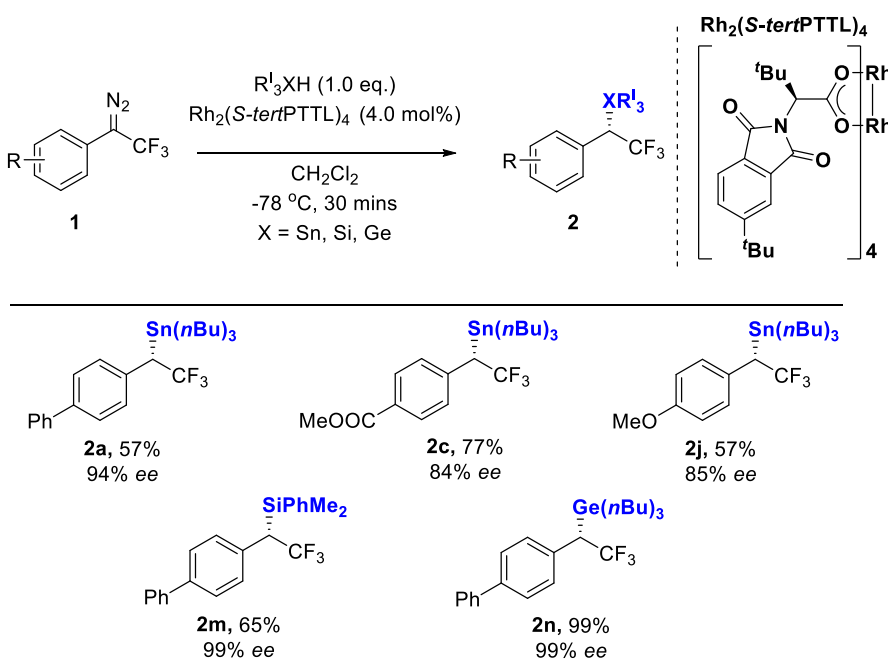

Scheme 3. Asymmetric rhodium-catalysed insertion into $\mathrm{X}-\mathrm{H}$ bonds ( $\mathrm{X}=\mathrm{Sn}$, $\mathrm{Ge}, \mathrm{Si})$ with trifluorodiazoethanes. Absolute configuration determined by comparison of $[\alpha]^{D}$ value of $\mathbf{2 m}$ with that reported in the literature. ${ }^{21}$ Absolute configuration of other substrates assigned by analogy with $2 \mathbf{m}$.

\section{Conclusion}

In summary, we have developed a Rh(II) catalysed trialkyltin hydride insertion reaction into 1-aryl-2,2,2-trifluorodiazoethanes. In contrast to products of Sn$H$ insertion of $\alpha$-carbonyl diazo compounds, the $\alpha$-(trifluoromethyl)benzyl stannanes are bench stable. In addition, the first asymmetric tin hydride insertion with diazo compounds has been disclosed delivering enantioenriched $\alpha$-(trifluoromethyl)benzyl stannanes. The use of the stannane products in palladium catalyzed cross coupling is challenging due to their propensity to undergo $\beta$-fluoride elimination. However, a recent report has demonstrated that secondary $\alpha$-(trifluoromethyl)benzyl tosylates can 
undergo cross coupling without $\beta$-fluoride elimination, suggesting that the same might possible for the corresponding stannanes disclosed within. ${ }^{31}$

\section{Experimental}

\subsection{General Information}

All NMR spectra were recorded on Bruker DPX200, AV400 or AV500 spectrometers and are reported as chemical shifts $(\delta)$ in parts per million (ppm). ${ }^{1} \mathrm{H}$ and ${ }^{13} \mathrm{C}$ NMR spectra are referenced relative to residual undeuterated solvent peak. ${ }^{19} \mathrm{~F}$ NMR spectra are referenced relative to $\mathrm{CFCl}_{3}$. Coupling constants $(\mathrm{J})$ are reported in units of hertz $(\mathrm{Hz})$. The following abbreviations are used to describe multiplets: $s$ (singlet), $d$ (doublet), $t$ (triplet), q (quartet), quint (quintuplet), sept (septet), m (multiplet), br (broad). High resolution mass spectra (HRMS, $\mathrm{m} / \mathrm{z}$ ) were recorded on a Bruker MicroTOF spectrometer using electrospray ionization (ESI), electron ionisation (EI) or chemical ionisation (CI). Infrared spectra were recorded as neat compound using a Bruker Tensor 27 FT-IR spectrometer. Absorptions are reported in wavenumbers $\left(\mathrm{cm}^{-1}\right)$ and only peaks of interest are reported. Melting points of solids were measured on a Griffin apparatus and are uncorrected. Thin layer chromatographies (TLC) were performed using Merck aluminium-foil baked plates precoated with Kieselgel 60 F254. The products were visualized using UV fluorescence $(254 \mathrm{~nm}$ ) or potassium permanganate stain. Flash column chromatography columns were performed over Merck silica gel C60 (40 $60 \mu \mathrm{m})$ using eluent systems as described for each experiment. All solvents and chemicals were purchased from Sigma-Aldrich, Acros, Alfa Aesar, Fluorochem or Apollo Scientific, and used without further purification, unless stated otherwise.

\subsection{Preparation of diazo substrates 1}

All diazo compounds $\mathbf{1}$ were prepared from the corresponding trifluoroacetophenones, either via general procedure $A$ followed by general procedure $\mathrm{B}$, or in a telescoped process using general procedure $\mathrm{C}$. The trifluoroacetophenones were prepared according to literature procedures. $^{23,32}$

\section{General procedure A: preparation of hydrazone diazo precursors 1'}

Procedure from Wang et al. ${ }^{33}$ To a round bottom flask equipped with a reflux condenser was added tosylhydrazide (1.0 eq.) and the minimum quantity of solvent (either methanol or toluene according to individual substrates) needed to dissolve the hydrazide at reflux (approximately $1.5 \mathrm{M}$ ). Subsequently the reaction was cooled to room temperature and trifluoroacetophenone (1.0 eq.) was added in one portion. The reaction mixture was then stirred at $65{ }^{\circ} \mathrm{C}(\mathrm{MeOH})$ or $90{ }^{\circ} \mathrm{C}$ (Toluene) over $4-16 \mathrm{~h}$ (monitored by TLC). The solution was cooled to room temperature or $0{ }^{\circ} \mathrm{C}$, at which point the product precipitated out of solution (precipitation can be induced by addition of pentane). The precipitate was collected by vacuum filtration and washed with pentane, in which case it was used without further purification. If no precipitation occurred, the solvent was removed under reduced pressure and the residue used in the next step without further purification. Hydrazone derivatives were found to be unstable on silica gel.

\section{General procedure B: preparation of diazo substrates 1}

CAUTION: diazo compounds are presumed to be toxic and potentially explosive and should be handled with care, in a fume hood. ${ }^{34}$ Procedure from $\mathrm{Xu}$ et al. ${ }^{35}$ The Tosyl hydrazone (1.0 eq.) was refluxed in a solution of $\mathrm{KOH}$ (2 eq.) in $\mathrm{MeOH}(0.4 \mathrm{M})$ for $1 \mathrm{~h}$ or until the colour of the solution no longer intensified. The reaction was then cooled to room temperature and diluted with water. The crude product was extracted with $\mathrm{CH}_{2} \mathrm{Cl}_{2}$ or pentane (see individual substrates for details), washed with saturated solutions of $\mathrm{NaHCO}_{3}$ and brine, dried with $\mathrm{MgSO}_{4}$, concentrated under reduced pressure, and purified by silica gel chromatography.

General procedure C: telescoped preparation of diazo substrates $\mathbf{1}$

CAUTION: diazo compounds are presumed to be toxic and potentially explosive and should be handled with care, in a fume hood. ${ }^{34}$ To a mixture of tosyl hydrazine (1.0 eq.) in $\mathrm{MeOH}(1.5 \mathrm{M})$ was added ketone (1.0 eq). The reaction mixture was heated to reflux for $18 \mathrm{~h}$ then allowed to cool to room temperature. Solvent was evaporated under reduced pressure and the residue triturated with pentane, dried under vacuum and used in subsequent steps without additional purification. To the residue was added $\mathrm{KOH}(2.0$ equiv.) in $\mathrm{MeOH}(0.4 \mathrm{M})$ and the reaction mixture heated to reflux for 1-2 hours, during which the characteristic red colour of diazo compounds appeared. The reaction mixture was allowed to cool to room temperature, poured into water and extracted with pentane $(x 3)$. The combined organic layers were dried over $\mathrm{Na}_{2} \mathrm{SO}_{4}$, filtered and evaporated under reduced pressure. The residue was purified by silica gel column chromatography.

\section{N'-(1-([1,1'-biphenyl]-4-yl)-2,2,2-trifluoroethylidene)-4-} methylbenzenesulfonohydrazide (1'a)

Prepared following general procedure $A$ from the corresponding trifluoroacetophenone $\left(6.7 \mathrm{~g}, 36 \mathrm{mmol}\right.$.), in toluene $\left(90^{\circ} \mathrm{C}, 12 \mathrm{~h}\right)$. Purified by precipitation upon cooling to room temperature and subsequent filtration. Obtained as an off-white solid (11.4 g, $76 \%$ ). $\left.{ }^{1} \mathrm{H} \mathrm{NMR} \mathrm{(400} \mathrm{MHz,} \mathrm{CDCl}_{3}\right) \delta 8.09$ $(\mathrm{s}, 1 \mathrm{H}), 7.84\left(\mathrm{~d},{ }^{3} \mathrm{~J}_{\mathrm{HH}}=8.3 \mathrm{~Hz}, 2 \mathrm{H}\right), 7.73\left(\mathrm{~d},{ }^{3} \mathrm{~J}_{\mathrm{HH}}=8.3 \mathrm{~Hz}, 2 \mathrm{H}\right), 7.60(\mathrm{~m}, 2 \mathrm{H}), 7.50$ $(\mathrm{m}, 2 \mathrm{H}), 7.43(\mathrm{~m}, 1 \mathrm{H}), 7.35(\mathrm{~m}, 3 \mathrm{H}), 2.47(\mathrm{~s}, 3 \mathrm{H}) .{ }^{13} \mathrm{C} \mathrm{NMR}\left(100 \mathrm{MHz}, \mathrm{CDCl}_{3}\right) \delta$ $145.2,144.7,141.6\left(q,{ }^{2} J_{\mathrm{CF}}=35.7 \mathrm{~Hz}\right), 139.7,134.7,130.1,129.3,128.9,128.8$, 128.6, 128.2, 127.4, 124.0, $120.2\left(\mathrm{q},{ }^{1} \mathrm{~J}_{\mathrm{CF}}=271 \mathrm{~Hz}\right), 21.9 .{ }^{19} \mathrm{~F} \mathrm{NMR}(376 \mathrm{MHz}$, $\left.\mathrm{CDCl}_{3}\right) \delta-68.1(\mathrm{~s}, 3 \mathrm{~F})$. Data consistent with literature values. ${ }^{23}$

\section{4-(1-diazo-2,2,2-trifluoroethyl)-1,1'-biphenyl (1a)}

Prepared following general procedure B from tosylhydrazone 1'a (7.41 g, 17.1 $\mathrm{mmol})$. Purified by silica gel chromatography (pentane) and obtained as a orange/red solid (1.86 g, $40 \%$ ). ${ }^{1} \mathrm{H}$ NMR $\left(400 \mathrm{MHz}, \mathrm{CDCl}_{3}\right) \delta 7.39(\mathrm{~m}, 2 \mathrm{H}), 7.29$ $(\mathrm{m}, 2 \mathrm{H}), 7.23(\mathrm{~m}, 2 \mathrm{H}), 7.14(\mathrm{~m}, 1 \mathrm{H}), 6.81\left(\mathrm{~d},{ }^{3} \mathrm{~J}_{\mathrm{HH}}=8.1 \mathrm{~Hz}, 2 \mathrm{H}\right) .{ }^{13} \mathrm{C}$ NMR $(126$ $\left.\mathrm{MHz}_{1} \mathrm{CDCl}_{3}\right) \delta 129.2,128.0,127.9,127.7,127.6,126.3\left(\mathrm{q},{ }^{1} \mathrm{~J}_{\mathrm{CF}}=270 \mathrm{~Hz}\right), 125.6$, 123.2, 122.1 ( $\mathrm{C}=\mathrm{N}_{2}$ not observed). ${ }^{19} \mathrm{~F} \mathrm{NMR}\left(376 \mathrm{MHz}, \mathrm{CDCl}_{3}\right) \delta-57.4(\mathrm{~s}, 3 \mathrm{~F})$. Data consistent with literature values. ${ }^{36}$

N'-(1-(4-bromophenyl)-2,2,2-trifluoroethylidene)-4methylbenzenesulfonohydrazide (1'b)

Prepared following general procedure A from 4'-bromo-2,2,2trifluoroacetophenone $\left(2.43 \mathrm{~g}, 9.6 \mathrm{mmol}\right.$.) in toluene $\left(90^{\circ} \mathrm{C}, 12 \mathrm{~h}\right)$. Purified by precipitation upon cooling to room temperature and subsequent filtration. Obtained as an off-white solid (3.72 g, 74\%). ${ }^{1} \mathrm{H}$ NMR $\left(400 \mathrm{MHz}, \mathrm{CDCl}_{3}\right) \delta 7.90$ $(\mathrm{s}, 1 \mathrm{H}) 7.81(\mathrm{~m}, 2 \mathrm{H}), 7.60(\mathrm{~m}, 2 \mathrm{H}), 7.36\left(\mathrm{~d},{ }^{3} \mathrm{~J}_{\mathrm{HH}}=7.8 \mathrm{~Hz}, 2 \mathrm{H}\right), 7.15\left(\mathrm{~d},{ }^{3} \mathrm{~J}_{\mathrm{HH}}=7.8\right.$ $\mathrm{Hz}, 2 \mathrm{H}), 2.47(\mathrm{~s}, 3 \mathrm{H}) .{ }^{13} \mathrm{C} \mathrm{NMR}\left(100 \mathrm{MHz}, \mathrm{CDCl}_{3}\right) \delta 145.3,140.5\left(\mathrm{q},{ }^{2} \mathrm{~J}_{\mathrm{CF}}=36.0\right.$ $\mathrm{Hz}), 134.5,133.7,130.1,130.0,128.4,126.8,124.3,120.0\left(\mathrm{q},{ }^{1} \mathrm{~J}_{\mathrm{CF}}=273 \mathrm{~Hz}\right.$ ), 21.9. ${ }^{19} \mathrm{~F} \mathrm{NMR}\left(376 \mathrm{MHz}, \mathrm{CDCl}_{3}\right) \delta-68.2(\mathrm{~s}, 3 \mathrm{~F})$. Data consistent with literature values. $^{33}$

\section{1-bromo-4-(1-diazo-2,2,2-trifluoroethyl)benzene (1b)}

Prepared following general procedure B from tosylhydrazone 1'b (3.5 g, 8.3 mmol.). Purified by silica gel chromatography (pentane) and obtained as a deep-red liquid (1.21 g, $55 \%)$. ${ }^{1} \mathrm{H}$ NMR (400 MHz, $\left.\mathrm{CDCl}_{3}\right) \delta 7.51(\mathrm{~m}, 2 \mathrm{H}), 6.97$ $\left(d,{ }^{3} J_{\mathrm{HH}}=8.6 \mathrm{~Hz}, 2 \mathrm{H}\right) \cdot{ }^{13} \mathrm{C} \mathrm{NMR}\left(100 \mathrm{MHz}, \mathrm{CDCl}_{3}\right) \delta 132.5,125.7\left(q,{ }^{1} \mathrm{JF}_{\mathrm{CF}}=270\right.$ 
$\mathrm{Hz}), 123.8,122.9,119.7$ ( $\mathrm{C}=\mathrm{N}_{2}$ not observed). $\left.{ }^{19} \mathrm{~F} \mathrm{NMR} \mathrm{(376} \mathrm{MHz}, \mathrm{CDCl}_{3}\right) \delta-$ $57.6(\mathrm{~s}, 3 \mathrm{~F})$. Data consistent with literature values. ${ }^{23}$

methyl-4-(2,2,2-trifluoro-1-(2-tosylhydrazineylidene)ethyl)benzoate (1'c)

Prepared following general procedure A from corresponding trifluoroacetophenone $\left(3.0 \mathrm{~g}, 12.9 \mathrm{mmol}\right.$.) in toluene $\left(90{ }^{\circ} \mathrm{C}, 12 \mathrm{~h}\right)$. Purified by precipitation upon cooling to room temperature and subsequent filtration. Obtained as an off-white solid (4.36 g, 85\%). $\left.{ }^{1} \mathrm{H} \mathrm{NMR} \mathrm{(400} \mathrm{MHz,} \mathrm{CDCl}_{3}\right) \delta 7.96$, $(\mathrm{s}, 1 \mathrm{H}), 7.84(\mathrm{~m}, 2 \mathrm{H}), 7.35(\mathrm{~m}, 4 \mathrm{H}), 7.15(\mathrm{~d}, 2 \mathrm{H}), 2.49(\mathrm{~s}, 3 \mathrm{H}), 2.40(\mathrm{~s}, 3 \mathrm{H}) .{ }^{13} \mathrm{C}$ NMR $\left(100 \mathrm{MHz}_{1} \mathrm{CDCl}_{3}\right) \delta 165.4,145.0,140.4\left(q,{ }^{2} \mathrm{~J}_{\mathrm{CF}}=36.5 \mathrm{~Hz}\right), 134.5,133.2$, $131.2,130.0,129.7,128.6,128.2,120.0\left(q,{ }^{1} \mathrm{~J}_{\mathrm{CF}}=273.0 \mathrm{~Hz}\right), 53.0,21.9 .{ }^{19} \mathrm{~F} N M R$ $\left(376 \mathrm{MHz} \mathrm{CDCl}_{3}\right) \delta-68.1$ (s, 3F). Data consistent with literature values. ${ }^{23}$

\section{methyl 4-(1-diazo-2,2,2-trifluoroethyl)benzoate (1c)}

Prepared following a modification of general procedure $\mathrm{B}$ : To a solution of tosylhydrazone 1'c (1.12 g, $2.8 \mathrm{mmol}$.) in $\mathrm{MeOH}(10 \mathrm{~mL})$ was added $\mathrm{NaOMe}$ (303 mg, 5.6 mmol., 2 eq.). A condenser was attached, and the reaction mixture refluxed for $1 \mathrm{~h}$. The reaction was cooled to room temperature and diluted with water. The crude product was extracted with $\mathrm{CH}_{2} \mathrm{Cl}_{2}$, washed with saturated solutions of $\mathrm{NaHCO}_{3}$ and brine, dried with $\mathrm{MgSO}_{4}$, concentrated under reduced pressure, and purified by silica gel chromatography (pentane $/ \mathrm{CH}_{2} \mathrm{Cl}_{2}$ 90:10) and obtained as an orange solid (414 $\mathrm{mg}, 63 \%$ ). ${ }^{1} \mathrm{H}$ $\operatorname{NMR}\left(400 \mathrm{MHz}, \mathrm{CDCl}_{3}\right) \delta 8.05\left(\mathrm{~d},{ }^{3} \mathrm{~J}_{\mathrm{HH}}=8.8 \mathrm{~Hz}, 2 \mathrm{H}\right), 6.73\left(\mathrm{~d},{ }^{3} \mathrm{~J}_{\mathrm{HH}}=8.8 \mathrm{~Hz}, 2 \mathrm{H}\right)$, $3.60(\mathrm{~s}, 3 \mathrm{H}) .{ }^{13} \mathrm{C} \mathrm{NMR}\left(126 \mathrm{MHz}, \mathrm{CDCl}_{3}\right) \delta 132.4,126.3,125.2$ (q, ${ }^{1} \mathrm{~J}_{\mathrm{CF}}=269.0$ $\mathrm{Hz}), 124.1,123.3,122.5,119.2\left(\mathrm{C}=\mathrm{N}_{2}\right.$ not observed). ${ }^{19} \mathrm{~F} \mathrm{NMR}\left(376 \mathrm{MHz}, \mathrm{CDCl}_{3}\right)$ $\delta-57.5(\mathrm{~s}, 3 \mathrm{~F})$. Data consistent with literature values. ${ }^{23}$

4-methyl-N'-(2,2,2-trifluoro-1-(p-tolyl)ethylidene)benzenesulfonohydrazide (1'd)

Prepared following general procedure A from 2,2,2-trifluoro-1-(p-tolyl)ethan1-one ( $1.0 \mathrm{~g}, 5.31 \mathrm{mmol}$.) in methanol $\left(65^{\circ} \mathrm{C}, 18 \mathrm{~h}\right)$. Purified by cooling to room temperature and subsequent filtration and washing with pentane. Obtained as a white solid $(0.73 \mathrm{~g}, 38 \%) .{ }^{1} \mathrm{H}$ NMR $\left(400 \mathrm{MHz}^{\mathrm{C}} \mathrm{CDCl}_{3}\right): \delta 7.95$ (s, $\left.1 \mathrm{H}\right) 7.81$ $\left(\mathrm{d},{ }^{3} \mathrm{~J}_{\mathrm{HH}}=8.3 \mathrm{~Hz}, 2 \mathrm{H}\right), 7.32(\mathrm{~m}, 4 \mathrm{H}), 7.13\left(\mathrm{~d},{ }^{3} \mathrm{~J}_{\mathrm{HH}}=8.3 \mathrm{~Hz}, 2 \mathrm{H}\right), 2.42(\mathrm{~s}, 3 \mathrm{H}), 2.47$ (s, 3H). ${ }^{13} \mathrm{C}$ NMR $\left(100 \mathrm{MHz}, \mathrm{CDCl}_{3}\right): \delta 144.9,142.1,141.8\left(\mathrm{q},{ }^{2} \mathrm{~J}_{\mathrm{CF}}=35.8 \mathrm{~Hz}\right)$, 134.6, 130.7, 129.9, 128.0, 122.1, $119.0\left(q,{ }^{1} \mathrm{~J}_{\mathrm{CF}}=275 \mathrm{~Hz}\right), 21.7,21.5\left(\mathrm{C}=\mathrm{N}_{2}\right.$ not observed). $\left.{ }^{19} \mathrm{~F} \mathrm{NMR} \mathrm{(376} \mathrm{MHz,} \mathrm{CDCl}_{3}\right) \delta-68.4$ (s, 3F). Data consistent with literature values. ${ }^{33}$

\section{1-(1-diazo-2,2,2-trifluoroethyl)-4-methylbenzene (1d)}

Prepared following general procedure B from tosylhydrazone 1'd $(0.72$ g, 2.0 $\mathrm{mmol}$ ), purified by silica gel chromatography (pentane) and obtained as a red liquid (169 mg, $42 \%) .{ }^{1} \mathrm{H} \mathrm{NMR}\left(400 \mathrm{MHz}, \mathrm{CDCl}_{3}\right) \delta 6.82\left(\mathrm{~d},{ }^{3} \mathrm{~J}_{\mathrm{HH}}=8.1 \mathrm{~Hz}, 2 \mathrm{H}\right)$, $6.75\left(\mathrm{~d}, 3^{3} \mathrm{HH}=8.1 \mathrm{~Hz}, 2 \mathrm{H}\right), 1.98(\mathrm{~s}, 3 \mathrm{H}) .{ }^{13} \mathrm{C} \mathrm{NMR}\left(126 \mathrm{MHz}, \mathrm{CDCl}_{3}\right) \delta 136.2$, $130.7,126.9\left(q,{ }^{1} \mathrm{CF}_{\mathrm{CF}}=270 \mathrm{~Hz}\right), 123.1,120.6,60.2\left(\mathrm{q},{ }^{2} \mathrm{~J}_{\mathrm{CF}}=43.9 \mathrm{~Hz}\right), 21.1 .{ }^{19} \mathrm{~F}$ NMR $\left(376 \mathrm{MHz}, \mathrm{CDCl}_{3}\right) \delta-57.5$ (s, 3F). Data consistent with literature values. ${ }^{23}$

\section{1-bromo-3-(1-diazo-2,2,2-trifluoroethyl)benzene (1e)}

Prepared following general procedure $C$ from 3'-bromo-2,2,2trifluoroacetophenone $(2.0 \mathrm{~g}, 7.9 \mathrm{mmol}$.). Purified by silica gel column chromatography (pentane) and obtained as a red oil (120 mg, $6 \%$ over two steps). ${ }^{1} \mathrm{H}$ NMR (500 MHz, $\left.\mathrm{C}_{6} \mathrm{D}_{6}\right): 7.02-6.88(\mathrm{~m}, 2 \mathrm{H}), 6.55(\mathrm{t}, \mathrm{J}=7.9 \mathrm{~Hz}, 1 \mathrm{H})$, $6.52-6.45(\mathrm{~m}, 1 \mathrm{H}) .{ }^{13} \mathrm{C}$ NMR $\left(125 \mathrm{MHz}, \mathrm{C}_{6} \mathrm{D}_{6}\right): \delta 130.6,128.7,125.8,125.5$ (q, $\left.{ }^{1} \mathrm{~J}_{\mathrm{CF}}=269.3 \mathrm{~Hz}\right), 124.7,123.5,120.4$, (C=N 2 not observed). ${ }^{19} \mathrm{~F} N \mathrm{NM}(471 \mathrm{MHz}$ $\left.\mathrm{C}_{6} \mathrm{D}_{6}\right) \delta-57.5$ (s, 3F). IR (neat) $\mathrm{u} / \mathrm{cm}^{-1}: 3071,2082,1594,1561,1481,1349$, $1324,1261,1172,1106,993,968,860,773,760,710,700,678 .{ }^{39}$
4-methyl-N'-(2,2,2-trifluoro-1-phenylethylidene)benzenesulfonohydrazide (1'f)

Prepared following general procedure A from trifluoroacetophenone $(2.09 \mathrm{~g}$, $12 \mathrm{mmol}$.), in methanol $\left(65^{\circ} \mathrm{C}, 12 \mathrm{~h}\right)$. Purified by precipitation upon cooling to room temperature and subsequent filtration. Obtained as an off-white solid (2.79 g, 68\%). ${ }^{1} \mathrm{H}$ NMR (400 MHz, $\left.\mathrm{CDCl}_{3}\right) \delta 7.92(\mathrm{~s}, 1 \mathrm{H}) 7.81(\mathrm{~m}, 2 \mathrm{H}), 7.50(\mathrm{~m}$, $3 \mathrm{H}), 7.35\left(\mathrm{~d},{ }^{3} \mathrm{~J}_{\mathrm{HH}}=8.0 \mathrm{~Hz}, 2 \mathrm{H}\right), 7.25(\mathrm{~m}, 2 \mathrm{H}), 2.47(\mathrm{~s}, 3 \mathrm{H}) .{ }^{13} \mathrm{C} \mathrm{NMR}(100 \mathrm{MHz}$, $\left.\mathrm{CDCl}_{3}\right) \delta 145.1,141.4\left(\mathrm{q},{ }^{2} \mathrm{~J}_{\mathrm{CF}}=35.7 \mathrm{~Hz}\right), 134.6,130.1,130.1,128.3,128.1$, 125.3, $120.1\left(\mathrm{q},{ }^{1} \mathrm{~J}_{\mathrm{CF}}=273 \mathrm{~Hz}\right), 21.8$ (unable to see $\mathrm{C}=\mathrm{N}_{2}$ ). ${ }^{19} \mathrm{~F}$ NMR (376 MHz, $\left.\mathrm{CDCl}_{3}\right) \delta-68.2(\mathrm{~s}, 3 \mathrm{~F})$. Data consistent literature values. ${ }^{22}$

\section{(1-diazo-2,2,2-trifluoroethyl)benzene (1f)}

Prepared following general procedure B from tosylhydrazone 1'f (2.91 g, 8.5 mmol.). Purified by silica gel chromatography (pentane) and obtained as a volatile red liquid (544 mg, $35 \%$ ). ${ }^{1} \mathrm{H}$ NMR $\left(400 \mathrm{MHz}, \mathrm{C}_{6} \mathrm{D}_{6}\right) \delta 7.42(\mathrm{~m}, 2 \mathrm{H}), 7.21$ $(\mathrm{m}, 1 \mathrm{H}), 7.12\left(\mathrm{~d},{ }^{3} \mathrm{~J}_{\mathrm{HH}}=8.2 \mathrm{~Hz}, 2 \mathrm{H}\right) .{ }^{13} \mathrm{C} N M R\left(126 \mathrm{MHz}, \mathrm{C}_{6} \mathrm{D}_{6}\right) \delta 130.0,126.7$ (q, $\left.{ }^{1} \mathrm{~J}_{\mathrm{CF}}=269 \mathrm{~Hz}\right), 126.5,123.9,122.9\left(\mathrm{C}=\mathrm{N}_{2}\right.$ not observed). ${ }^{19} \mathrm{~F} \mathrm{NMR}(376 \mathrm{MHz}$, $\left.\mathrm{C}_{6} \mathrm{D}_{6}\right) \delta-57.3(\mathrm{~s}, 3 \mathrm{~F})$. Data consistent with literature values. ${ }^{22}$

4-methyl-N'-(2,2,2-trifluoro-1-(4-

fluorophenyl)ethylidene)benzenesulfonohydrazide (1'g)

Prepared following general procedure A from 2,2,2,4' tetrafluoroacetophenone $\left(1.0 \mathrm{~g}, 5.20 \mathrm{mmol}\right.$.) in methanol $\left(65^{\circ} \mathrm{C}, 12 \mathrm{~h}\right)$. Purified by cooling to room temperature and subsequent filtration and washing with pentane. Obtained as a white solid (1.45 g, $77 \%)$. Used without further purification. ${ }^{1} \mathrm{H} N M R\left(400 \mathrm{MHz} \mathrm{CDCl}_{3}\right): \delta 7.98(\mathrm{~s}, 1 \mathrm{H}), 7.80(\mathrm{~d}, \mathrm{~J}=8.3$ $\mathrm{Hz}, 2 \mathrm{H}), 7.36(\mathrm{~d}, \mathrm{~J}=8.0 \mathrm{~Hz}, 2 \mathrm{H}), 7.29-7.19(\mathrm{~m}, 4 \mathrm{H}), 2.46(\mathrm{~s}, 3 \mathrm{H}) .{ }^{13} \mathrm{C} N M R(101$ $\mathrm{MHz}_{\mathrm{CDCl}}$ ): $\delta 164.2\left(\mathrm{~d},{ }^{1} \mathrm{~J}_{\mathrm{CF}}=252.7 \mathrm{~Hz}\right), 145.2,134.5,130.9,130.8,130.0$, $128.2,120.1\left(\mathrm{q},{ }^{1} \mathrm{~J}_{\mathrm{CF}}=274.9 \mathrm{~Hz}\right), 117.7,117.5,21.8 .{ }^{19} \mathrm{~F} \mathrm{NMR}\left(377 \mathrm{MHz}, \mathrm{CDCl}_{3}\right)$ : $\delta-68.4(\mathrm{~s}, 3 \mathrm{~F}),-106.6(\mathrm{~m}, 1 \mathrm{~F})$. Data consistent with literature values. ${ }^{33}$

\section{1-(1-diazo-2,2,2-trifluoroethyl)-4-fluorobenzene (1g)}

Prepared following general procedure B from tosylhydrazone 1'g (1.45 g, 4.0 $\mathrm{mmol})$. Purified by silica gel chromatography (pentane) and obtained as a orange/red liquid (250 mg, $31 \%$ ). ${ }^{1} \mathrm{H}$ NMR $\left(400 \mathrm{MHz}, \mathrm{CDCl}_{3}\right): \delta$ 7.14-7.06 (m, $4 \mathrm{H}) .{ }^{13} \mathrm{C}$ NMR (101 MHz, CDCl 3 ): $\delta 161.7\left(\mathrm{~d},{ }^{1} \mathrm{~J}_{\mathrm{CF}}=246.8 \mathrm{~Hz}\right), 126.0\left(\mathrm{q},{ }^{1} \mathrm{~J}_{\mathrm{CF}}=\right.$ $269.2 \mathrm{~Hz}$ ), $124.4(\mathrm{dq}, \mathrm{J}=8.1,1.4 \mathrm{~Hz}), 119.4(\mathrm{~d}, \mathrm{~J}=3.5 \mathrm{~Hz}), 116.8$ (d, J = 22.3 $\mathrm{Hz}),\left(\mathrm{C}=\mathrm{N}_{2}\right.$ not observed). ${ }^{19} \mathrm{~F} \mathrm{NMR}\left(376 \mathrm{MHz}^{\mathrm{C} C D C l}\right.$ ) $): \delta-57.6(\mathrm{~s}, 3 \mathrm{~F}),-115.9(\mathrm{~s}$, 1F). IR (neat) U/ cm ${ }^{-1}: 3054,2080,1512,1334,1306,1270,1240,1167,1099$, $957,825,735,609$. HRMS (ESI, negative) calculated for $\mathrm{C}_{8} \mathrm{H}_{3} \mathrm{~F}_{4} \mathrm{~N}_{2}-([\mathrm{M}-\mathrm{H}]-)$ : 203.0238, observed: 203.0233 .

\section{1-(1-diazo-2,2,2-trifluoroethyl)-4-(trifluoromethyl)benzene (1h)}

Prepared following general procedure C from 4'-trifluoromethyl-2,2,2trifluoroacetophenone (1.01 g, $4.2 \mathrm{mmol}$.). Purified by silica gel column chromatography (pentane) and obtained as a yellow oil (46 mg, $4 \%$ over two steps). ${ }^{1} \mathrm{H} N M R\left(500 \mathrm{MHz}, \mathrm{CDCl}_{3}\right): \delta 7.73-7.50(\mathrm{~m}, 2 \mathrm{H}), 7.22-7.07(\mathrm{~m}, 2 \mathrm{H}) .{ }^{13} \mathrm{C}$ $\operatorname{NMR}\left(125 \mathrm{MHz}_{\mathrm{CDCl}}\right.$ ): $\delta 128.3,128.0\left(\mathrm{q},{ }^{2} \mathrm{~J}_{\mathrm{CF}}=32.9 \mathrm{~Hz}\right), 126.5(\mathrm{q}, \mathrm{J}=3.9 \mathrm{~Hz})$, $125.2\left(q,{ }^{1} \mathrm{JFF}_{\mathrm{CF}}=269.7 \mathrm{~Hz}\right), 124.0\left(\mathrm{q},{ }^{1} \mathrm{~J}_{\mathrm{CF}}=271.8 \mathrm{~Hz}\right), 122.0(\mathrm{~d}, \mathrm{~J}=1.0 \mathrm{~Hz}),\left(\mathrm{C}=\mathrm{N}_{2}\right.$ not observed). ${ }^{19} \mathrm{~F} \mathrm{NMR} \mathrm{(376} \mathrm{MHz,} \mathrm{CDCl}_{3}$ ): $\delta-57.4$ (s, 3F), -62.6 (s, 3F). IR (neat) $\mathrm{u} / \mathrm{cm}^{-1}$ : 2931, 2859, 2088, 1619, 1523, 1325, 1280, 1206, 1169, 1110, 1069, $1016,958,831,738 .{ }^{39}$

\section{2-chloro-4-(1-diazo-2,2,2-trifluoroethyl)-1-fluorobenzene (1i)}

Prepared following general procedure $\mathrm{C}$ from the corresponding trifluoroacetophenone $(1.0 \mathrm{~g}, 4.4 \mathrm{mmol})$. Purified by silica gel column chromatography (pentane) and obtained as a red oil (14 mg, $2 \%$ over two 
steps). ${ }^{1} \mathrm{H} \mathrm{NMR}\left(400 \mathrm{MHz}, \mathrm{CDCl}_{3}\right): \delta 7.19(\mathrm{t}, \mathrm{J}=8.6 \mathrm{~Hz}, 1 \mathrm{H}), 7.13(\mathrm{dd}, \mathrm{J}=6.4$, $2.4 \mathrm{~Hz}, 1 \mathrm{H}), 6.98-6.94(\mathrm{~m}, 1 \mathrm{H}) .{ }^{13} \mathrm{C} \mathrm{NMR}\left(101 \mathrm{MHz}, \mathrm{CDCl}_{3}\right): \delta 156.6\left(\mathrm{~d},{ }^{1} \mathrm{~J} \mathrm{CF}=\right.$ $249.8 \mathrm{~Hz}), 126.7,125.6\left(\mathrm{q},{ }^{1} \mathrm{~J}_{\mathrm{CF}}=269.4 \mathrm{~Hz}\right), 124.6(\mathrm{~d}, \mathrm{~J}=1.3 \mathrm{~Hz}), 122.3(\mathrm{~m})$, $121.1(\mathrm{~d}, \mathrm{~J}=4.2 \mathrm{~Hz}), 117.9(\mathrm{~d}, \mathrm{~J}=22.0 \mathrm{~Hz}),\left(\mathrm{C}=\mathrm{N}_{2}\right.$ not observed). ${ }^{19} \mathrm{~F}$ NMR $(376$ $\mathrm{MHz}, \mathrm{CDCl}_{3}$ ): $\delta-57.5$ (s, 3F), -118.1 (td, J $=7.2,4.2 \mathrm{~Hz}, 1 \mathrm{~F}$ ). IR (neat) $\mathrm{v} / \mathrm{cm}^{-1}$ : 2925, 2853, 2237, 2172, 2089, 2011, 1599, 1504, 1466, 1324, 1257, 1194, $1145,1066,814,704,628,617 .^{39}$

4-methyl-N'-(2,2,2-trifluoro-1-(4-

methoxyphenyl)ethylidene)benzenesulfonohydrazide (1'j)

Prepared following general procedure A from 4'-methoxy-2,2,2trifluoroacetophenone $\left(4.08 \mathrm{~g}, 20 \mathrm{mmol}\right.$.) in methanol $\left(65^{\circ} \mathrm{C}, 12 \mathrm{~h}\right)$. Purified by precipitation upon cooling to room temperature and subsequent filtration. Obtained as a pale-yellow solid $(2.31 \mathrm{~g}, 28 \%) .{ }^{1} \mathrm{H}$ NMR $\left(400 \mathrm{MHz}, \mathrm{CD}_{3} \mathrm{OD}\right) \delta$ 7.80-7.79 (m, 2H), 7.43 (m, 2H), 7.32-7.30 (m, 2H), 7.09-7.06 (m, 2H), $3.87(\mathrm{~s}$, $3 \mathrm{H}), 2.45(\mathrm{~s}, 3 \mathrm{H}$ ), (unable to see $\mathrm{N}-\mathrm{H}) .{ }^{13} \mathrm{C}$ NMR $\left(100 \mathrm{MHz}, \mathrm{CD}_{3} \mathrm{OD}\right) \delta 161.5$, $144.1,141.0\left(\mathrm{q},{ }^{2} \mathrm{~J}_{\mathrm{CF}}=38.9 \mathrm{~Hz}\right), 135.3,129.8,129.2,127.7,120.4\left(\mathrm{q},{ }^{1} \mathrm{~J}_{\mathrm{CF}}=274\right.$ $\mathrm{Hz}), 118.9,114.2,54.2,20.0 .{ }^{19} \mathrm{~F} \mathrm{NMR} \mathrm{(376} \mathrm{MHz,} \mathrm{CD}_{3} \mathrm{OD}$ ) $\delta-65.5$ (s, 3F). Data consistent with literature values. ${ }^{23}$

\section{1-(1-diazo-2,2,2-trifluoroethyl)-4-methoxybenzene (1j)}

Prepared following general procedure B from tosylhydrazone $\mathbf{1}^{\prime} \mathbf{j}(2.0 \mathrm{~g}, 5.38$ mmol.). Purified by basic alumina gel chromatography (hexane) and obtained as a red solid (139 mg, $12 \%) .{ }^{1} \mathrm{H} \mathrm{NMR}\left(500 \mathrm{MHz}, \mathrm{C}_{6} \mathrm{D}_{6}\right) \delta$ 6.75-6.73 $(\mathrm{m}, 2 \mathrm{H})$, $6.60\left(\mathrm{~d},{ }^{3} \mathrm{~J}_{\mathrm{HH}}=9.0 \mathrm{~Hz}, 2 \mathrm{H}\right), 3.21(\mathrm{~s}, 3 \mathrm{H}) .{ }^{19} \mathrm{~F} \mathrm{NMR}\left(376 \mathrm{MHz}, \mathrm{CDCl}_{3}\right) \delta-57.6(\mathrm{~s}$, 3F). Data consistent with literature values. ${ }^{37}$

\section{4-(1-diazo-2,2,2-trifluoroethyl)-2-fluoro-1-methylbenzene (1k)}

Prepared following general procedure $\mathrm{C}$ from the corresponding trifluoroacetophenone $(1.8 \mathrm{~g}, 8.7 \mathrm{mmol}$.). Purified by silica gel column chromatography (pentane) and obtained as a red oil (47 $\mathrm{mg}, 2 \%$ over two steps). ${ }^{1} \mathrm{H}$ NMR (500 MHz, $\left.\mathrm{CD}_{2} \mathrm{Cl}_{2}\right): \delta 7.24(\mathrm{t}, \mathrm{J}=8.2 \mathrm{~Hz}, 1 \mathrm{H}), 6.86-6.71(\mathrm{~m}$, $2 \mathrm{H}), 2.26\left(\mathrm{~d},{ }^{4} \mathrm{~J}_{\mathrm{HF}}=1.9 \mathrm{~Hz}, 3 \mathrm{H}\right) \cdot{ }^{13} \mathrm{C}$ NMR $(126 \mathrm{MHz}$, Methylene Chloride-d2) $\delta$ $162.4(\mathrm{~d}, \mathrm{~J}=244.9 \mathrm{~Hz}), 133.1(\mathrm{~d}, \mathrm{~J}=6.1 \mathrm{~Hz}), 126.2(\mathrm{q}, \mathrm{J}=269.0 \mathrm{~Hz}), 123.4$ (d, J $=9.1 \mathrm{~Hz}), 123.2(\mathrm{~d}, \mathrm{~J}=17.3 \mathrm{~Hz}), 118.3(\mathrm{~d}, \mathrm{~J}=3.3 \mathrm{~Hz}), 109.6(\mathrm{~d}, \mathrm{~J}=26.2 \mathrm{~Hz}), 14.4$ $\left(\mathrm{C}=\mathrm{N}_{2}\right.$ not observed). ${ }^{19} \mathrm{~F} \mathrm{NMR}\left(376 \mathrm{MHz}, \mathrm{CDCl}_{3}\right): \delta-56.0(\mathrm{~s}, 3 \mathrm{~F}),-114.3(\mathrm{~m}, 1 \mathrm{~F})$. IR (neat) U/ cm cm $^{-1}$ 2960, 2933, 2867, 2082, 1629, 1579, 1514, 1354, 1327, 1295, $1273,1235,1204,1104,1009,875,862,807,760,734,644$. Compound 1k was found to be unstable under various ionisation techniques and HRMS could therefore not be obtained.

4-methyl-N'-(2,2,2-trifluoro-1-(4-

propylphenyl)ethylidene)benzenesulfonohydrazide (1'I)

Prepared following general procedure A from 4'-N-propyl-2,2,2trifluoroacetophenone $\left(1.0 \mathrm{~g}, 4.60 \mathrm{mmol}\right.$.) in methanol $\left(65^{\circ} \mathrm{C}, 12 \mathrm{~h}\right)$. Purified by cooling to room temperature and subsequent filtration and washing with pentane. Obtained as a white solid $(1.33 \mathrm{~g}, 75 \%)$. Used without further purification. ${ }^{1} \mathrm{H} \mathrm{NMR}(400 \mathrm{MHz}, \mathrm{CDCl} 3): \delta 7.98(\mathrm{~s}, 1 \mathrm{H}), 7.81(\mathrm{~d}, \mathrm{~J}=8.3 \mathrm{~Hz}, 2 \mathrm{H})$, $7.367 .31(\mathrm{~m}, 4 \mathrm{H}), 7.14(\mathrm{~d}, \mathrm{~J}=8.0 \mathrm{~Hz}, 2 \mathrm{H}), 2.63\left(\mathrm{t},{ }^{3} \mathrm{~J}_{\mathrm{HH}}=7.7 \mathrm{~Hz}, 2 \mathrm{H}\right), 2.46(\mathrm{~s}$, $3 \mathrm{H}), 1.71,1.62(\mathrm{~m}, 2 \mathrm{H}), 0.97\left(\mathrm{t},{ }^{3} \mathrm{~J}_{\mathrm{HH}}=7.3 \mathrm{~Hz}, 3 \mathrm{H}\right) .{ }^{13} \mathrm{C} \mathrm{NMR}\left(126 \mathrm{MHz} \mathrm{CDCl}_{3}\right)$ : $\delta 146.9,145.0,142.0\left(q,{ }^{2} \mathrm{~J}_{\mathrm{CF}}=35.6 \mathrm{~Hz}\right), 134.7,130.2,130.0,128.1,122.5$, $120.1\left(\mathrm{q},{ }^{1} \mathrm{~J}_{\mathrm{CF}}=274.9 \mathrm{~Hz}\right), 77.2,38.1,24.3,21.8,14.0 .{ }^{19} \mathrm{~F} \mathrm{NMR}(377 \mathrm{MHz}$, $\mathrm{CDCl}_{3}$ ): $\delta-68.3(\mathrm{~s}, 3 \mathrm{~F})$. MP 108-109 ${ }^{\circ} \mathrm{C}$. IR (neat) u/ $\mathrm{cm}^{-1}: 3171,2964,2924$, 2857, 1359, 1170, 1119, 1070, 999, 811, 684. HRMS (ESI, positive) calculated for $\mathrm{C}_{18} \mathrm{H}_{20} \mathrm{~F}_{3} \mathrm{~N}_{2} \mathrm{O}_{2} \mathrm{~S}^{+}\left([\mathrm{M}+\mathrm{H}]^{+}\right)$: 385.1192 , observed: 385.1202.
Prepared following general procedure B from tosylhydrazone 1'l (1.33 g, 3.46 $\mathrm{mmol})$, purified by silica gel chromatography (pentane) and obtained as a orange/red liquid (194 mg, $25 \%$ ). ${ }^{1} \mathrm{H} \mathrm{NMR}\left(400 \mathrm{MHz}, \mathrm{CDCl}_{3}\right): \delta 7.23\left(\mathrm{~d},{ }^{3} \mathrm{~J}_{\mathrm{HH}}=\right.$ $8.6 \mathrm{~Hz}, 2 \mathrm{H}), 7.03\left(\mathrm{~d},{ }^{3} \mathrm{~J}_{\mathrm{HH}}=7.9 \mathrm{~Hz}, 2 \mathrm{H}\right), 2.60\left(\mathrm{t},{ }^{3} \mathrm{~J}_{\mathrm{HH}}=7.6 \mathrm{~Hz}, 2 \mathrm{H}\right), 1.70-1.61(\mathrm{~m}$, $2 \mathrm{H}), 0.96(\mathrm{t}, 3 \mathrm{JHH}=7.3 \mathrm{~Hz}, 3 \mathrm{H}) .{ }^{19} \mathrm{~F} \mathrm{NMR}\left(377 \mathrm{MHz}^{\mathrm{C} C D C l}\right): \delta-57.4(\mathrm{~s}, 3 \mathrm{~F}) .{ }^{13} \mathrm{C}$ $\operatorname{NMR}\left(101 \mathrm{MHz}, \mathrm{CDCl}_{3}\right): \delta 140.9,129.7,125.7\left(\mathrm{q},{ }^{1} \mathrm{~J}_{\mathrm{CF}}=268.6 \mathrm{~Hz}\right), 122.5,120.5$, 37.6, 24.6, 13.9, ( $C=\mathrm{N}_{2}$ not observed). IR (neat) $\mathrm{U} / \mathrm{cm}^{-1}: 2963,2934,2874$, 2079, 1516, 1345, 1276, 1167, 1145, 1105, 957, 835, 799, 738, 640. HRMS (ESI, negative) calculated for $\mathrm{C}_{11} \mathrm{H}_{10} \mathrm{~F}_{3} \mathrm{~N}_{2}{ }^{-}([\mathrm{M}-\mathrm{H}]-)$ : 227.0802 , observed: 227.0556 .

\subsection{Diazo insertion reaction}

General procedure D: $\mathrm{CF}_{3} \mathrm{C}(\mathrm{Ar}) \mathrm{N}_{2}$ Insertion into $n \mathrm{Bu}_{3} \mathrm{SnH}$ under inert atmosphere

A flame dried, argon purged vial was charged with aryl 2,2,2trifluorodiazoethane 1 (1.0 eq., $0.1 \mathrm{mmol}$ ), $n \mathrm{Bu} 3 \mathrm{SnH}(26.5 \mu \mathrm{L}, 1.0 \mathrm{eq}, 0.1$ mmol) and degassed $\mathrm{CH}_{2} \mathrm{Cl}_{2}(0.7 \mathrm{~mL})$. In a separate flame dried, argon purged vial was added $\mathrm{Rh}_{2}(\mathrm{OAc})_{4}$ (1.8 mg, $4 \mathrm{~mol} \%, 0.004 \mathrm{mmol}$ ) and degassed $\mathrm{CH}_{2} \mathrm{Cl}_{2}$ $(0.5 \mathrm{~mL})$. Diazo compound and $n \mathrm{Bu}_{3} \mathrm{SnH}$ solution were added to the $\mathrm{Rh}_{2}(\mathrm{OAc})_{4}$ suspension over $<1 \mathrm{~min}$ at room temperature. After evolution of $\mathrm{N}_{2}$ had stopped and characteristic orange/red colour of diazo compound had disappeared, the reaction progress was monitored by TLC. Typically, the reaction was complete in less than 15 minutes. The reaction mixture was filtered through a short plug of silica gel eluting with $\mathrm{CH}_{2} \mathrm{Cl}_{2}$, evaporated under reduced pressure and the crude product purified by silica gel column chromatography.

General procedure $\mathrm{E}: \mathrm{CF}_{3} \mathrm{C}(\mathrm{Ar}) \mathrm{N}_{2}$ Insertion into $n \mathrm{Bu}_{3} \mathrm{SnH}$ in an open vial

To a solution of aryl 2,2,2-trifluorodiazoethane 1 (1.0 eq, $0.1 \mathrm{mmol}$ ) and $n \mathrm{Bu}_{3} \mathrm{SnH}(26.5 \mu \mathrm{L}, 1.0 \mathrm{eq}, 0.1 \mathrm{mmol})$ in $\mathrm{CH}_{2} \mathrm{Cl}_{2}(1.2 \mathrm{~mL})$ in an open vial was added $\mathrm{Rh}_{2}(\mathrm{OAc})_{4}(1.77 \mathrm{mg}, 4 \mathrm{~mol} \%, 0.004 \mathrm{mmol})$. After evolution of $\mathrm{N}_{2}$ had stopped and characteristic orange/red colour of diazo compound had disappeared, the reaction progress was monitored by TLC. Typically, the reaction was complete in less than $15 \mathrm{~min}$. The reaction mixture was filtered through a short plug of silica gel eluting with $\mathrm{CH}_{2} \mathrm{Cl}_{2}$, evaporated under reduced pressure and the crude product purified by silica gel column chromatography.

General Procedure F: Asymmetric $\mathrm{CF}_{3} \mathrm{C}(\mathrm{Ar}) \mathrm{N}_{2}$ insertion into $n \mathrm{Bu} 3 \mathrm{SnH}$

A flame dried, argon purged vial was charged with aryl 2,2,2trifluorodiazoethane 1 (1.0 eq, $0.1 \mathrm{mmol}), n \mathrm{Bu}_{3} \mathrm{SnH}(26.5 \mu \mathrm{L}, 1.0 \mathrm{eq}, 0.1 \mathrm{mmol})$ and degassed $\mathrm{CH}_{2} \mathrm{Cl}_{2}(0.7 \mathrm{~mL})$. In a separate flame dried, argon purged vial was added $\mathrm{Rh}_{2}$ (S-tertPTTL) 4 (1.77 mg, $4 \mathrm{~mol} \%, 0.004 \mathrm{mmol}$ ) and degassed $\mathrm{CH}_{2} \mathrm{Cl}_{2}$ $(0.5 \mathrm{~mL})$ and the mixture was cooled down to $-78^{\circ} \mathrm{C}$. The diazo compound and $n \mathrm{Bu}_{3} \mathrm{SnH}$ solution were added to the $\mathrm{Rh}_{2}(\mathrm{~S} \text {-tertPTTL })_{4}$ suspension over $<1 \mathrm{~min}$ at $-78{ }^{\circ} \mathrm{C}$. After evolution of $\mathrm{N}_{2}$ had stopped and characteristic orange/red colour of diazo compound had disappeared, the reaction progress was monitored by TLC. Typically, the reaction was complete in less than $30 \mathrm{~min}$. The reaction mixture was filtered through a short plug of silica gel eluting with $\mathrm{CH}_{2} \mathrm{Cl}_{2}$, evaporated under reduced pressure and the crude product was purified by silica gel column chromatography.

\section{(1-([1,1'-biphenyl]-4-yl)-2,2,2-trifluoroethyl)tributylstannane (2a)}

Prepared following general procedure D. Purified by silica gel column chromatography (pentane) and obtained as a colourless oil (39 mg, $74 \%$ ). ${ }^{1} \mathrm{H}$ $\operatorname{NMR}\left(400 \mathrm{MHz}, \mathrm{CDCl}_{3}\right): \delta$ 7.60-7.58 $(\mathrm{m}, 2 \mathrm{H}), 7.53(\mathrm{~d}, \mathrm{~J}=8.4 \mathrm{~Hz}, 2 \mathrm{H}), 7.44(\mathrm{t}, \mathrm{J}$ $=7.6 \mathrm{~Hz}, 2 \mathrm{H}), 7.34(\mathrm{t}, \mathrm{J}=7.4 \mathrm{~Hz}, 1 \mathrm{H}), 7.22(\mathrm{~d}, \mathrm{~J}=8.2 \mathrm{~Hz}, 2 \mathrm{H}), 3.23\left(\mathrm{q},{ }^{3} \mathrm{~J}_{\mathrm{HF}}=13.7\right.$ $\mathrm{Hz}, 1 \mathrm{H}), 1.47-1.39(\mathrm{~m}, 6 \mathrm{H}), 1.28(\mathrm{~m}, 6 \mathrm{H}), 0.97(\mathrm{~m}, 6 \mathrm{H}), 0.87\left(\mathrm{t},{ }^{3} \mathrm{~J}_{\mathrm{HH}}=7.3 \mathrm{~Hz}\right.$, 
9H). ${ }^{13} \mathrm{C} \mathrm{NMR} \mathrm{(101} \mathrm{MHz,} \mathrm{CDCl} 3$ ): $\delta 140.9,138.7,135.8$ (q, J = 3.8 Hz), 129.7 (q, $\left.{ }^{1} \mathrm{~J}_{\mathrm{CF}}=274.3 \mathrm{~Hz}\right), 128.9,128.3,127.5,127.3,127.0,38.9(\mathrm{q}, 2 \mathrm{JCF}=30.9 \mathrm{~Hz})$, $28.8,27.4,13.7,10.6 .{ }^{19} \mathrm{~F} \mathrm{NMR}\left(376 \mathrm{MHz}, \mathrm{CDCl}_{3}\right): \delta-55.7\left(\mathrm{~d},{ }^{3} \mathrm{~J}_{\mathrm{FH}}=13.6 \mathrm{~Hz}, 3 \mathrm{~F}\right)$. IR (neat) u/ cm $\mathrm{cm}^{-1}$ : 3031, 2956, 2923, 2872, 2854, 1907, 1729, 1612, 1600, 1519, $1488,1463,1416,1377,1345,1306,1281,1261,1238,1204,1123,1101$, $1075,1039,1008,960,912,878,848,823,764,740,727,695,668,614$. HRMS (Cl) calculated for $\mathrm{C}_{14} \mathrm{H}_{10} \mathrm{~F}_{2}{ }^{+}\left(\left[\mathrm{M}-n \mathrm{nu}_{3} \mathrm{SnF}\right]^{+}\right): 216.0751$, observed: 216.0753 ; calculated for $\mathrm{C}_{12} \mathrm{H}_{27} \mathrm{Sn}^{+}\left(\left[n \mathrm{Bu}_{3} \mathrm{Sn}\right]^{+}\right)$: 291.1129, observed: $291.1145 .{ }^{38}$ Asymmetric reaction was performed using general procedure $\mathrm{F}$. The product (+)-(2a) was obtained in $57 \%$ yield with this procedure. $94 \%$ ee, HPLC conditions; Chiralpak IA column, hexane $=100 \%$, flow rate $=1.0 \mathrm{~mL} / \mathrm{min}, 25$ ${ }^{\circ} \mathrm{C}$, wavelength $=254 \mathrm{~nm}, \mathrm{tR}=5.89 \mathrm{~min}$ for minor isomer, $\mathrm{tR}=6.72 \mathrm{~min}$ for major isomer: $[\alpha]^{\mathrm{D}} 25=+55.7$ (c $1.46, \mathrm{MeOH}$ ).

\section{(1-(4-bromophenyl)-2,2,2-trifluoroethyl)tributylstannane (2b)}

Prepared following general procedure E. Purified by silica gel column chromatography (pentane) and obtained as a colourless oil (34 mg, $64 \%$ ). ${ }^{1} \mathrm{H}$ NMR $\left(400 \mathrm{MHz}, \mathrm{CDCl}_{3}\right): \delta 7.40(\mathrm{~d}, \mathrm{~J}=8.6 \mathrm{~Hz}, 2 \mathrm{H}), 7.01(\mathrm{~d}, \mathrm{~J}=8.4 \mathrm{~Hz}, 2 \mathrm{H}), 3.12$ $\left(q,{ }^{3} J_{\mathrm{HF}}=13.6 \mathrm{~Hz}, 1 \mathrm{H}\right), 1.45-1.37(\mathrm{~m}, 6 \mathrm{H}), 1.29-1.24(\mathrm{~m}, 6 \mathrm{H}), 0.99-0.90(\mathrm{~m}, 6 \mathrm{H})$, $0.87\left(\mathrm{t},{ }^{3} \mathrm{~J}_{\mathrm{HH}}=7.3 \mathrm{~Hz}, 9 \mathrm{H}\right) .{ }^{13} \mathrm{C} \mathrm{NMR}\left(101 \mathrm{MHz}, \mathrm{CDCl}_{3}\right): \delta 135.9,131.9,130.7$ (q, $\left.{ }^{1} \mathrm{~J}_{\mathrm{CF}}=275.0 \mathrm{~Hz}\right), 129.5,119.6,38.7\left(\mathrm{q},{ }^{2} \mathrm{~J}_{\mathrm{CF}}=31.2 \mathrm{~Hz}\right), 28.7,27.4,13.7,10.5 .{ }^{19} \mathrm{~F}$ $\operatorname{NMR}\left(376 \mathrm{MHz}, \mathrm{CDCl}_{3}\right): \delta-55.9\left(\mathrm{~d},{ }^{3} \mathrm{~J}_{\mathrm{FH}}=13.5 \mathrm{~Hz}, 3 \mathrm{~F}\right) . \mathrm{IR}$ (neat) $\mathrm{u} / \mathrm{cm}^{-1}: 2957$, 2924, 2872, 2854, 1895, 1731, 1592, 1490, 1463, 1412, 1377, 1350, 1339, $1301,1264,1238,1196,1127,1100,1076,1039,1011,960,941,875,843$, $809,769,747,721,691,671,649$. HRMS (Cl) calculated for $\mathrm{C}_{12} \mathrm{H}_{27} \mathrm{Sn}^{+}$ $\left(\left[n B u_{3} S n\right]^{+}\right): 291.1129$, observed: $291.1138 .{ }^{38}$

\section{methyl 4-(2,2,2-trifluoro-1-(tributylstannyl)ethyl)benzoate (2c)}

Prepared following general procedure E. Purified by silica gel column chromatography (100\% pentane to pentane/ $\mathrm{CH}_{2} \mathrm{Cl}_{2} 1: 1$ ) and obtained as a colourless oil ( $31 \mathrm{mg}, 61 \%) .{ }^{1} \mathrm{H} \mathrm{NMR}\left(400 \mathrm{MHz}, \mathrm{CDCl}_{3}\right): \delta 7.95\left(\mathrm{~d},{ }^{3} \mathrm{~J}_{\mathrm{HH}}=8.5\right.$ $\mathrm{Hz}, 2 \mathrm{H}), 7.19\left(\mathrm{~d},{ }^{3} \mathrm{~J}_{\mathrm{HH}}=8.3 \mathrm{~Hz}, 2 \mathrm{H}\right), 3.90(\mathrm{~s}, 3 \mathrm{H}), 3.25\left(\mathrm{q},{ }^{3} \mathrm{~J}_{\mathrm{HF}}=13.5 \mathrm{~Hz}, 1 \mathrm{H}\right), 1.45-$ $1.31(\mathrm{~m}, 6 \mathrm{H}), 1.25(\mathrm{~m}, 6 \mathrm{H}), 1.01-0.89(\mathrm{~m}, 6 \mathrm{H}), 0.85\left(\mathrm{t},{ }^{3} \mathrm{~J}_{\mathrm{HH}}=7.3 \mathrm{~Hz}, 9 \mathrm{H}\right) .{ }^{13} \mathrm{C}$ $\operatorname{NMR}\left(126 \mathrm{MHz}, \mathrm{CDCl}_{3}\right): \delta 167.0,142.5(\mathrm{q}, \mathrm{J}=4.0 \mathrm{~Hz}), 130.2,129.3\left(\mathrm{q},{ }^{1} \mathrm{~J}_{\mathrm{CF}}=\right.$ $275.0 \mathrm{~Hz}), 127.5,127.4,52.2,39.7\left(\mathrm{q},{ }^{2} \mathrm{~J}_{\mathrm{CF}}=31.2 \mathrm{~Hz}\right), 28.7,27.4,13.7,10.6 .{ }^{19} \mathrm{~F}$ $\operatorname{NMR}\left(377 \mathrm{MHz}, \mathrm{CDCl}_{3}\right): \delta \quad-55.3\left(\mathrm{~d},{ }^{3} \mathrm{~J}_{\mathrm{FH}}=13.2 \mathrm{~Hz}, 3 \mathrm{~F}\right) . \mathrm{IR}$ (neat) $\mathrm{U} / \mathrm{cm}^{-1}: 2956$, 2925, 2873, 2855, 1724, 1610, 1436, 1348, 1275, 1239, 1184, 1112, 1021, 962, 860, 771, 711. HRMS (Cl) calculated for $\mathrm{C}_{22} \mathrm{H}_{36} \mathrm{~F}_{3} \mathrm{O}_{2} \mathrm{Sn}^{+}\left([\mathrm{M}+\mathrm{H}]^{+}\right)$: 509.1689 , observed: 509.1677. Asymmetric reaction was performed using general procedure $\mathrm{F}$. The product $(+)-(\mathbf{2 c})$ was obtained in $77 \%$ yield with this procedure. $84 \%$ ee, HPLC conditions; Chiralpak IA column, hexane $=100 \%$, flow rate $=1.0 \mathrm{~mL} / \mathrm{min}, 25^{\circ} \mathrm{C}$, wavelength $=254 \mathrm{~nm}, \mathrm{tR}=10.50 \mathrm{~min}$ for major isomer, $t R=12.13 \mathrm{~min}$ for minor isomer: $[\alpha]^{\mathrm{D}}{ }_{25}=+1.0(\mathrm{c} 1.26, \mathrm{MeOH})$.

\section{tributyl(2,2,2-trifluoro-1-(p-tolyl)ethyl)stannane (2d)}

Prepared following general procedure D. Purified by silica gel column chromatography (pentane) and obtained as a colourless oil (30 mg, $65 \%) .{ }^{1} \mathrm{H}$ NMR $\left(500 \mathrm{MHz}, \mathrm{CDCl}_{3}\right): \delta 7.08(\mathrm{~d}, \mathrm{~J}=8.0 \mathrm{~Hz}, 2 \mathrm{H}), 7.03(\mathrm{~d}, \mathrm{~J}=8.1 \mathrm{~Hz}, 2 \mathrm{H}), 3.12$ $\left(\mathrm{q},{ }^{3} \mathrm{~J}_{\mathrm{HF}}=13.8 \mathrm{~Hz}, 1 \mathrm{H}\right), 2.31(\mathrm{~s}, 3 \mathrm{H}), 1.47-1.32(\mathrm{~m}, 6 \mathrm{H}), 1.26(\mathrm{~m}, 6 \mathrm{H}), 0.99-0.90$ $(\mathrm{m}, 6 \mathrm{H}), 0.86\left(\mathrm{t},{ }^{3} \mathrm{~J}_{\mathrm{HH}}=7.3 \mathrm{~Hz}, 9 \mathrm{H}\right) .{ }^{13} \mathrm{C} \mathrm{NMR}\left(126 \mathrm{MHz}, \mathrm{CDCl}_{3}\right): \delta 135.4,133.4$ $(q, J=3.9 \mathrm{~Hz}), 129.7\left(q,{ }^{1} \mathrm{~J}_{\mathrm{CF}}=275.0 \mathrm{~Hz}\right), 129.5,127.9,38.7\left(\mathrm{q},{ }^{2} \mathrm{~J}_{\mathrm{CF}}=30.8 \mathrm{~Hz}\right)$, 28.8, 27.4, 21.1, 13.7, 10.4. ${ }^{19} \mathrm{~F} \mathrm{NMR}\left(376 \mathrm{MHz}, \mathrm{CDCl}_{3}\right): \delta-56.0\left(\mathrm{~d},{ }^{3} \mathrm{JFH}_{\mathrm{FH}}=13.7\right.$ $\mathrm{Hz}, 3 F$ ). IR (neat) U/ cm ${ }^{-1}: 2957,2923,2872,2855,1513,1463,1342,1309$, $1241,1199,1123,1103,1074,1042,876,808,719.43,665$. HRMS (CI) calculated for $\mathrm{C}_{9} \mathrm{H}_{8} \mathrm{~F}_{2}^{+}\left(\left[\mathrm{M}-n \mathrm{Bu}_{3} \mathrm{SnF}\right]^{+}\right)$: 154.0594, observed: 154.0585; calculated for $\mathrm{C}_{12} \mathrm{H}_{27} \mathrm{Sn}^{+}\left(\left[n \mathrm{Bu}_{3} \mathrm{Sn}\right]^{+}\right)$: 291.1129, observed: $291.1129 .{ }^{38}$

(1-(3-bromophenyl)-2,2,2-trifluoroethyl)tributylstannane (2e)
Prepared following general procedure D. Purified by silica gel column chromatography (pentane) and obtained as a colourless oil (31 mg, 59\%). ${ }^{1} \mathrm{H}$ NMR (500 MHz, $\left.\mathrm{CDCl}_{3}\right)$ : $\delta$ 7.31-7.29 (m, 2H), $7.15(\mathrm{t}, \mathrm{J}=8.1 \mathrm{~Hz}, 1 \mathrm{H}), 7.07$ (d, J $=7.9 \mathrm{~Hz}, 1 \mathrm{H}), 3.12\left(\mathrm{q},{ }^{3} \mathrm{~J}_{\mathrm{HF}}=13.5 \mathrm{~Hz}, 1 \mathrm{H}\right), 1.45-1.34(\mathrm{~m}, 6 \mathrm{H}), 1.27(\mathrm{~m}, 6 \mathrm{H}), 1.01-$ $0.90(\mathrm{~m}, 6 \mathrm{H}), 0.87\left(\mathrm{t},{ }^{3} \mathrm{~J}_{\mathrm{HH}}=7.3 \mathrm{~Hz}, 9 \mathrm{H}\right) .{ }^{13} \mathrm{C} \mathrm{NMR}\left(126 \mathrm{MHz} \mathrm{CDCl}_{3}\right): \delta 139.3-$ $139.1(\mathrm{~m}), 130.7,130.3,129.3\left(\mathrm{q},{ }^{1} \mathrm{~J}_{\mathrm{CF}}=275.0 \mathrm{~Hz}\right), 128.9,126.3,122.9,38.9$ (q, $\left.{ }^{2} \mathrm{~J}_{\mathrm{CF}}=31.2 \mathrm{~Hz}\right), 28.7,27.4,13.7,10.6 .{ }^{19} \mathrm{~F} \mathrm{NMR}\left(377 \mathrm{MHz} \mathrm{CDCl}_{3}\right): \delta-55.7\left(\mathrm{~d},{ }^{3} \mathrm{~J}_{\mathrm{FH}}\right.$ $=13.4 \mathrm{~Hz}, 3 \mathrm{~F}$ ). IR (neat) $\mathrm{u} / \mathrm{cm}^{-1}: 2957,2923,2872,2854,1593,1563,1475$, $1378,1341,1239,1195,1128,1088,1039,996,877,779,713,692,620$. HRMS (Cl) calculated for $\mathrm{C}_{12} \mathrm{H}_{27} \mathrm{Sn}^{+}\left(\left[n \mathrm{Bu}_{3} \mathrm{Sn}\right]^{+}\right)$: 291.1129, observed: $291.1132 .^{38}$

\section{tributyl(2,2,2-trifluoro-1-phenylethyl)stannane (2f)}

Prepared following general procedure D. Purified by silica gel column chromatography (pentane) and obtained as a colourless oil (22 $\mathrm{mg}, 49 \%) .{ }^{1} \mathrm{H}$ NMR $\left(500 \mathrm{MHz}, \mathrm{CDCl}_{3}\right)$ : $\delta 7.26(\mathrm{~s}, 2 \mathrm{H}), 7.17-7.13(\mathrm{~m}, 3 \mathrm{H}), 3.16\left(\mathrm{q},{ }^{3} \mathrm{~J}_{\mathrm{HF}}=13.7\right.$ $\mathrm{Hz}, 1 \mathrm{H}), 1.43-1.36(\mathrm{~m}, 6 \mathrm{H}), 1.26(\mathrm{~m}, 6 \mathrm{H}), 0.97-0.90(\mathrm{~m}, 6 \mathrm{H}), 0.86\left(\mathrm{t}, 3^{3} \mathrm{HH}=7.3\right.$ $\mathrm{Hz}, 9 \mathrm{H}) .{ }^{13} \mathrm{CNMR}\left(126 \mathrm{MHz}, \mathrm{CDCl}_{3}\right): \delta 136.6$ (q, J = 3.9 Hz), $129.7\left(\mathrm{q},{ }^{1} \mathrm{~J}_{\mathrm{CF}}=274.9\right.$ $\mathrm{Hz}), 128.8,127.9,125.8,39.2\left(\mathrm{q},{ }^{2} \mathrm{~J}_{\mathrm{CF}}=30.9 \mathrm{~Hz}\right), 28.7,27.4,13.7,10.5 .{ }^{19} \mathrm{~F} N \mathrm{NMR}$ (377 MHz, CDCl 3 ): $\delta-55.8\left(d,{ }^{3} \mathrm{~J}_{\mathrm{FH}}=13.5 \mathrm{~Hz}, 3 \mathrm{~F}\right.$ ). IR (neat) U/ $\mathrm{cm}^{-1}: 2957,2924$, 2872, 2854, 1601, 1495, 1456, 1356, 1277, 1240, 1203, 1125, 1074, 1034, 874, $757,698,671,615$. HRMS (Cl) calculated for $\mathrm{C}_{8} \mathrm{H}_{6} \mathrm{~F}_{2}{ }^{+}\left(\left[\mathrm{M}-n \mathrm{Bu}_{3} \mathrm{SnF}\right]^{+}\right): 140.0438$ observed: 140.0426; calculated for $\mathrm{C}_{12} \mathrm{H}_{27} \mathrm{Sn}^{+}\left(\left[n \mathrm{Bu}_{3} \mathrm{Sn}\right]^{+}\right): 291.1129$, observed: $291.1135 . .^{38}$

\section{tributyl(2,2,2-trifluoro-1-(4-fluorophenyl)ethyl)stannane (2g)}

Prepared following general procedure D. Purified by silica gel column chromatography (pentane) and obtained as a colourless oil (25 mg, $54 \%$ ). ${ }^{1} \mathrm{H}$ $\operatorname{NMR}\left(500 \mathrm{MHz}, \mathrm{CDCl}_{3}\right): \delta 7.10$ (dd, J = 8.4, $\left.5.4 \mathrm{~Hz}, 2 \mathrm{H}\right), 6.98(\mathrm{t}, \mathrm{J}=8.7 \mathrm{~Hz}, 2 \mathrm{H})$, $3.13\left(\mathrm{q},{ }^{3} \mathrm{~J}_{\mathrm{HF}}=13.6 \mathrm{~Hz}, 1 \mathrm{H}\right), 1.46-1.34(\mathrm{~m}, 6 \mathrm{H}), 1.26(\mathrm{~m}, 6 \mathrm{H}), 1.00-0.89(\mathrm{~m}, 6 \mathrm{H})$, $0.86\left(\mathrm{t},{ }^{3} \mathrm{~J}_{\mathrm{HH}}=7.3 \mathrm{~Hz}, 9 \mathrm{H}\right) .{ }^{13} \mathrm{C} \mathrm{NMR}\left(126 \mathrm{MHz}, \mathrm{CDCl}_{3}\right): \delta 161.2\left(\mathrm{~d},{ }^{1} \mathrm{~J}_{\mathrm{CF}}=244.5\right.$ $\mathrm{Hz}), 132.4(q, J=3.9 \mathrm{~Hz}), 129.5\left(\mathrm{q},{ }^{1} \mathrm{~J}_{\mathrm{CF}}=274.9 \mathrm{~Hz}\right), 129.5(\mathrm{q}, \mathrm{J}=7.8 \mathrm{~Hz}), 115.6$ $(d, J=21.3 \mathrm{~Hz}), 38.3\left(q,{ }^{2} \mathrm{JFF}_{\mathrm{CF}} 31.2 \mathrm{~Hz}\right), 28.7,27.4,13.7,10.5 .{ }^{19} \mathrm{~F}$ NMR $(471$ $\mathrm{MHz}, \mathrm{CDCl}_{3}$ ): $\delta-56.4\left(\mathrm{~d},{ }^{3} \mathrm{~J}_{\mathrm{FH}}=3.6 \mathrm{~Hz}, 3 \mathrm{~F}\right),-117.3(\mathrm{~m}, 1 \mathrm{~F}) . \mathrm{IR}$ (neat) $\mathrm{u} / \mathrm{cm}^{-1}$ : 2958, 2924, 2873, 2855, 1607, 1509, 1464, 1420, 1342, 1272, 1246, 1228, 1200, 1161, 1117, 1094, 1042, 961, 876, 825, 791, 721, 664. HRMS (Cl) calculated for $\mathrm{C}_{8} \mathrm{H}_{5} \mathrm{~F}_{3}{ }^{+}\left(\left[\mathrm{M}-n \mathrm{Bu}_{3} \mathrm{SnF}\right]^{+}\right)$: 158.0343 observed: 158.0330; calculated for $\mathrm{C}_{12} \mathrm{H}_{27} \mathrm{Sn}^{+}\left(\left[n \mathrm{Bu}_{3} \mathrm{Sn}\right]^{+}\right)$: 291.1129, observed: $291.1133 .^{38}$

\section{tributyl(2,2,2-trifluoro-1-(4-(trifluoromethyl)phenyl)ethyl)stannane (2h)}

Prepared following general procedure D. Purified by silica gel column chromatography (pentane) and obtained as a colourless oil (32 mg, 62 \%). ${ }^{1} \mathrm{H}$ NMR (400 MHz, CDCl $)$ : $\delta 7.54(\mathrm{~d}, \mathrm{~J}=8.1 \mathrm{~Hz}, 2 \mathrm{H}), 7.24(\mathrm{~d}, \mathrm{~J}=8.2 \mathrm{~Hz}, 2 \mathrm{H}), 3.24$ $\left(\mathrm{q},{ }^{3} \mathrm{~J}_{\mathrm{HF}}=13.4 \mathrm{~Hz}, 1 \mathrm{H}\right), 1.49-1.32(\mathrm{~m}, 6 \mathrm{H}), 1.32-1.16(\mathrm{~m}, 6 \mathrm{H}), 1.02-0.90(\mathrm{~m}$, $6 \mathrm{H}), 0.86\left(\mathrm{t},{ }^{3} \mathrm{~J}_{\mathrm{HH}}=7.3 \mathrm{~Hz}, 9 \mathrm{H}\right) .{ }^{13} \mathrm{C} \mathrm{NMR}\left(101 \mathrm{MHz} \mathrm{CDCl}_{3}\right): \delta 141.0,129.1(\mathrm{q}$, $\left.{ }^{1} \mathrm{~J}_{\mathrm{CF}}=271.8 \mathrm{~Hz}\right), 127.8\left(\mathrm{q},{ }^{2} \mathrm{~J}_{\mathrm{CF}}=32.7 \mathrm{~Hz}\right), 127.6(\mathrm{t}, \mathrm{J}=1.4 \mathrm{~Hz}), 125.6(q, \mathrm{~J}=3.5$ $\mathrm{Hz}), 124.2\left(\mathrm{q},{ }^{1} \mathrm{~J}_{\mathrm{CF}}=271.8 \mathrm{~Hz}\right), 39.2\left(\mathrm{q},{ }^{2} \mathrm{~J}_{\mathrm{CF}}=31.3 \mathrm{~Hz}\right), 28.5,27.2,13.5,10.4 .{ }^{19} \mathrm{~F}$ NMR (376 MHz, CDCl$): ~ \delta-55.5\left(d,{ }^{3} \mathrm{JHH}_{\mathrm{FH}}=13.4 \mathrm{~Hz}, 3 \mathrm{~F}\right),-62.4(3 \mathrm{~F})$. IR (neat) u/ $\mathrm{cm}^{-1}: 2959,2925,2874,2856,1620,1582,1519,1464,1423,1378,1326$, 1286, 1240, 1202, 1166, 1121, 1103, 1069, 1019, 960, 909, 879, 849, 825, 767, 742 , 690, 671. HRMS (Cl) calculated for $\mathrm{C}_{12} \mathrm{H}_{27} \mathrm{Sn}^{+}\left(\left[n \mathrm{Bu}_{3} \mathrm{Sn}\right]^{+}\right)$: 291.1129, observed: $291.1129 .{ }^{38}$

tributyl(1-(3-chloro-4-fluorophenyl)-2,2,2-trifluoroethyl)stannane (2i)

Prepared following general procedure $D$, on $0.05 \mathrm{mmol}$ scale. Purified by silica gel column chromatography (pentane) and obtained as a colourless oil (13 mg, $52 \%) .{ }^{1} \mathrm{H}$ NMR $\left(500 \mathrm{MHz}, \mathrm{CDCl}_{3}\right): \delta 7.17(\mathrm{dd}, \mathrm{J}=6.9,2.3 \mathrm{~Hz}, 1 \mathrm{H}), 7.06(\mathrm{t}, \mathrm{J}=$ $8.7 \mathrm{~Hz}, 1 \mathrm{H}), 7.00-6.97(\mathrm{~m}, 1 \mathrm{H}), 3.09\left(\mathrm{q},{ }^{3} \mathrm{~J}_{\mathrm{HF}}=13.4 \mathrm{~Hz}, 1 \mathrm{H}\right), 1.44-1.38(\mathrm{~m}, 6 \mathrm{H})$, 1.30-1.23 (m, 6H), 0.99-0.92 (m, 6H), $0.87\left(\mathrm{t}, 1 \mathrm{JHH}^{\mathrm{HH}}=7.3 \mathrm{~Hz}, 9 \mathrm{H}\right) .{ }^{13} \mathrm{C}$ NMR $(126$ $\left.\mathrm{MHz}_{\mathrm{CDCl}}\right): \delta 156.5\left(\mathrm{~d},{ }^{1} \mathrm{~J}_{\mathrm{CF}}=247.8 \mathrm{~Hz}\right), 134.1-133.8(\mathrm{~m}), 129.8,129.3\left(\mathrm{q},{ }^{1} \mathrm{~J}_{\mathrm{CF}}\right.$ 
$=275.0 \mathrm{~Hz}$ ), $127.5(\mathrm{~d}, \mathrm{~J}=7.0 \mathrm{~Hz}), 121.2(\mathrm{~d}, \mathrm{~J}=17.9 \mathrm{~Hz}), 116.8(\mathrm{~d}, \mathrm{~J}=21.0 \mathrm{~Hz})$, $38.2\left(q,{ }^{2} J_{C F}=31.5 \mathrm{~Hz}\right), 28.7,27.4,13.7,10.6 .{ }^{19} \mathrm{~F} \mathrm{NMR}\left(377 \mathrm{MHz}, \mathrm{CDCl}_{3}\right): \delta-$ $56.2\left(d,{ }^{3} J_{F H}=13.2 \mathrm{~Hz}, 3 F\right),-119.6-(m, 1 F) . I R$ (neat) $\mathrm{v} / \mathrm{cm}^{-1}: 2958,2924,2855$, $1499,1236,1230,1109$. HRMS $(\mathrm{Cl})$ calculated for $\mathrm{C}_{8} \mathrm{H}_{4} \mathrm{ClF}_{3}{ }^{+}\left(\left[\mathrm{M}-n \mathrm{Bu} \mathrm{u}_{3} \mathrm{SnF}\right]^{+}\right)$: 191.9954 observed: 191.9974; calculated for $\mathrm{C}_{12} \mathrm{H}_{27} \mathrm{Sn}^{+}\left(\left[n \mathrm{Bu}_{3} \mathrm{Sn}\right]^{+}\right)$: 291.1129, observed: $291.1146 .{ }^{38}$

\section{tributyl(2,2,2-trifluoro-1-(4-methoxyphenyl)ethyl)stannane (2j)}

Prepared following general procedure D. Purified by preparative TLC (5\% EtOAc in cyclohexane) and obtained as a colourless oil (18.3 mg, $38 \%) .{ }^{1} \mathrm{H}$ NMR (500 MHz, $\left.\mathrm{CDCl}_{3}\right)$ : $\delta$ 7.11-7.02 (m, 2H), 6.87-6.79 (m, 2H), $3.79(\mathrm{~s}, 3 \mathrm{H})$, $3.09\left(\mathrm{q},{ }^{3} \mathrm{~J}_{\mathrm{HF}}=13.8 \mathrm{~Hz}, 1 \mathrm{H}\right), 1.491 .30(\mathrm{~m}, 6 \mathrm{H}), 1.26(\mathrm{~h}, \mathrm{~J}=7.3 \mathrm{~Hz}, 6 \mathrm{H}), 1.02$ $0.87(\mathrm{~m}, 6 \mathrm{H}), 0.86\left(\mathrm{t},{ }^{3} \mathrm{~J}_{\mathrm{HH}}=7.3 \mathrm{~Hz}, 9 \mathrm{H}\right) .{ }^{13} \mathrm{C} \mathrm{NMR}\left(125 \mathrm{MHz}, \mathrm{CDCl}_{3}\right): \delta 157.9$, $129.8\left(q,{ }^{1} J_{C F}=274.9 \mathrm{~Hz}\right), 129.3,128.5(q, \mathrm{~J}=4.2 \mathrm{~Hz}), 114.2,55.4,38.1\left(q,{ }^{2} \mathrm{~J}_{\mathrm{CF}}\right.$ $=30.9 \mathrm{~Hz}), 28.8,27.4,13.7,10.4 .{ }^{19} \mathrm{~F} \mathrm{NMR}\left(376 \mathrm{MHz}, \mathrm{CDCl}_{3}\right): \delta-56.5\left(\mathrm{~d},{ }^{3} \mathrm{~J}_{\mathrm{FH}}=\right.$ $13.6 \mathrm{~Hz}, 3 F$ ). IR (neat) U/ cm${ }^{-1}:$ 2956, 2924, 2872, 2854, 1612, 1581, 1511 , $1464,1443,1423,1377,1351,1307,1283,1250,1235,1202,1180,1122$, $1100,1075,1038,961,931,875,849,821,775,747,726,687,665$. HRMS (CI) calculated for $\mathrm{C}_{9} \mathrm{H}_{8} \mathrm{~F}_{2} \mathrm{O}^{+}\left(\left[\mathrm{M}-n \mathrm{Bu}_{3} \mathrm{SnF}\right]^{+}\right)$: 170.0543, observed: 170.0520; calculated for $\mathrm{C}_{12} \mathrm{H}_{27} \mathrm{Sn}^{+}\left(\left[\mathrm{nBu}_{3} \mathrm{Sn}\right]^{+}\right)$: 291.1129, observed: 291.1116. ${ }^{38}$ Asymmetric reaction was performed using general procedure $\mathrm{F}$. The product (-)-(2j) was obtained in $57 \%$ yield with this procedure. $85 \%$ ee, HPLC conditions; Chiralpak IC column, hexane $=100 \%$, flow rate $=1.0 \mathrm{~mL} / \mathrm{min}, 25$ ${ }^{\circ} \mathrm{C}$, wavelength $=254 \mathrm{~nm}, \mathrm{tR}=9.20 \mathrm{~min}$ for major isomer, $\mathrm{tR}=10.11 \mathrm{~min}$ for minor isomer: $[\alpha]^{\mathrm{D}_{25}}=-0.6(\mathrm{c} \quad 0.46, \mathrm{MeOH})$.

\section{tributyl(2,2,2-trifluoro-1-(3-fluoro-4-methylphenyl)ethyl)stannane (2k)}

Prepared following general procedure D. Purified by silica gel column chromatography (pentane) and obtained as a colourless oil (14.5 mg, $60 \%$ ). ${ }^{1} \mathrm{H} \mathrm{NMR}\left(500 \mathrm{MHz} \mathrm{CDCl}_{3}\right): \delta 7.13-7.02(\mathrm{~m}, 1 \mathrm{H}), 6.88-6.74(\mathrm{~m}, 2 \mathrm{H}), 3.11\left(\mathrm{q},{ }^{3} \mathrm{~J}_{\mathrm{HF}}\right.$ $=13.6 \mathrm{~Hz}, 1 \mathrm{H}), 2.23(\mathrm{~d}, \mathrm{~J}=1.9 \mathrm{~Hz}, 3 \mathrm{H}), 1.50-1.34(\mathrm{~m}, 6 \mathrm{H}), 1.34-1.13(\mathrm{~m}, 6 \mathrm{H})$, $1.04-0.89(\mathrm{~m}, 6 \mathrm{H}), 0.86\left(\mathrm{t},{ }^{3} \mathrm{~J}_{\mathrm{HH}}=7.2 \mathrm{~Hz}, 9 \mathrm{H}\right) .{ }^{13} \mathrm{C} \mathrm{NMR}\left(125 \mathrm{MHz}, \mathrm{CDCl}_{3}\right): \delta$ $161.4\left(\mathrm{~d},{ }^{1} \mathrm{~J}_{\mathrm{CF}}=244.6 \mathrm{~Hz}\right), 136.2(\mathrm{dd}, \mathrm{J}=7.8,3.9 \mathrm{~Hz}), 131.7(\mathrm{~d}, \mathrm{~J}=5.8 \mathrm{~Hz}), 129.5$ $\left(q,{ }^{1} J_{C F}=274.8 \mathrm{~Hz}\right), 123.3(d, J=3.1 \mathrm{~Hz}), 122.1(\mathrm{~d}, \mathrm{~J}=17.2 \mathrm{~Hz}), 114.4(\mathrm{~d}, \mathrm{~J}=23.2$ $\mathrm{Hz}$ ), 39.2-38.2 (m), 28.7, 27.4, $14.3(\mathrm{~d}, \mathrm{~J}=3.3 \mathrm{~Hz}), 13.7,10.5 .{ }^{19} \mathrm{~F}$ NMR (376 $\mathrm{MHz}, \mathrm{CDCl}_{3}$ ): $\delta-56.0\left(\mathrm{~d}, 3_{\mathrm{FH}}=13.5 \mathrm{~Hz}, 3 \mathrm{~F}\right.$ ), -117.2 (ddd, J = 10.6, 8.4, 2.2 Hz, 1F). IR (neat) U/ cm ${ }^{-1}: 2957,2925,2873,2855,627,1579,1510,1463,1425$, 1378, 1340, 1290, 1272, 1238, 1207, 1150, 1128, 1075, 1041, 999, 958, 876, $851,817,787,755,710,683$. HRMS $(\mathrm{Cl})$ calculated for $\mathrm{C}_{9} \mathrm{H}_{7} \mathrm{~F}_{3}{ }^{+}\left(\left[\mathrm{M}-n \mathrm{Bu}_{3} \mathrm{SnF}\right]^{+}\right)$: 172.0500, observed: 173.0472; calculated for $\mathrm{C}_{12} \mathrm{H}_{27} \mathrm{Sn}^{+}\left(\left[n \mathrm{Bu}_{3} \mathrm{Sn}\right]^{+}\right): 291.1129$, observed: $291.1122 .{ }^{38}$

\section{tributyl(2,2,2-trifluoro-1-(4-propylphenyl)ethyl)stannane (2I)}

Prepared following general procedure D. Purified by silica gel column chromatography (pentane) and obtained as a colourless oil (25 mg, $51 \%) .{ }^{1} \mathrm{H}$ NMR $\left(500 \mathrm{MHz}, \mathrm{CDCl}_{3}\right): \delta 7.08(\mathrm{~d}, \mathrm{~J}=8.2 \mathrm{~Hz}, 2 \mathrm{H}), 7.04(\mathrm{~d}, \mathrm{~J}=8.2 \mathrm{~Hz}, 2 \mathrm{H}), 3.13$ $\left(\mathrm{q},{ }^{3} \mathrm{~J}_{\mathrm{HF}}=13.8 \mathrm{~Hz}, 1 \mathrm{H}\right), 2.55(\mathrm{t}, \mathrm{J}=7.6 \mathrm{~Hz}, 2 \mathrm{H}), 1.62(\mathrm{~m}, 2 \mathrm{H}), 1.45-1.31(\mathrm{~m}, 6 \mathrm{H})$, $1.25(\mathrm{~m}, 6 \mathrm{H}), 0.980 .89(\mathrm{~m}, 9 \mathrm{H}), 0.85\left(\mathrm{t},{ }^{3} \mathrm{~J}_{\mathrm{HH}}=7.3 \mathrm{~Hz}, 9 \mathrm{H}\right) .{ }^{13} \mathrm{C} \mathrm{NMR}(126 \mathrm{MHz}$, $\left.\mathrm{CDCl}_{3}\right): \delta 140.2,133.6(\mathrm{q}, \mathrm{J}=3.9 \mathrm{~Hz}), 129.8\left(\mathrm{q},{ }^{1} \mathrm{~J}_{\mathrm{CF}}=274.8 \mathrm{~Hz}\right), 128.8,127.9$, $38.7\left(q,{ }^{2} J_{C F}=30.7 \mathrm{~Hz}\right), 37.7,28.8,27.4,24.6,13.9,13.7,10.4 .{ }^{19} \mathrm{~F}$ NMR $(377$ $\mathrm{MHz}, \mathrm{CDCl}_{3}$ ): $\delta-56.0\left(\mathrm{~d},{ }^{3} \mathrm{~J}_{\mathrm{FH}}=13.8 \mathrm{~Hz}, 3 \mathrm{~F}\right.$ ). IR (neat) $\mathrm{U} / \mathrm{cm}^{-1}: 2958,2926,2872$, $2855,1512,1463,1343,1240,1198,1125,1106,1041,877,668$. HRMS (Cl) calculated for $\mathrm{C}_{11} \mathrm{H}_{12} \mathrm{~F}_{2}{ }^{+}\left(\left[\mathrm{M}-n \mathrm{Bu}_{3} \mathrm{SnF}\right]^{+}\right)$: 182.0907, observed: 182.0905 ; calculated for $\mathrm{C}_{12} \mathrm{H}_{27} \mathrm{Sn}^{+}\left(\left[n \mathrm{Bu}_{3} \mathrm{Sn}\right]^{+}\right)$: 291.1129, observed: $291.1136 .{ }^{38}$

\section{(1-([1,1'-biphenyl]-4-yl)-2,2,2-trifluoroethyl)dimethyl(phenyl)silane (2m)}

Prepared following general procedure $\mathrm{D}$, with substitution of $n \mathrm{Bu}_{3} \mathrm{SnH}$ for $\mathrm{PhMe}_{2} \mathrm{SiH}(15.3 \mu \mathrm{L}, 1.0 \mathrm{eq}, 0.1 \mathrm{mmol})$. Purified by silica gel column

chromatography (5-15\% $\mathrm{CH}_{2} \mathrm{Cl}_{2}$ in pentane) and obtained as a white solid in $92 \%$ yield (34 mg, $0.09 \mathrm{mmol}) .{ }^{1} \mathrm{H}$ NMR $\left(400 \mathrm{MHz}, \mathrm{CDCl}_{3}\right) \delta 7.58(\mathrm{~d}, \mathrm{~J}=7.3 \mathrm{~Hz}$, $2 \mathrm{H}), 7.49-7.41(\mathrm{~m}, 4 \mathrm{H}), 7.40-7.30(\mathrm{~m}, 6 \mathrm{H}), 7.06(\mathrm{~d}, \mathrm{~J}=8.1 \mathrm{~Hz}, 2 \mathrm{H}), 3.16(\mathrm{q}, \mathrm{J}=$ $\left.12.9 \mathrm{~Hz}, 1 \mathrm{H}), 0.48(\mathrm{~s}, 3 \mathrm{H}), 0.40(\mathrm{~s}, 3 \mathrm{H}) .{ }^{13} \mathrm{C} \mathrm{NMR} \mathrm{(126} \mathrm{MHz}, \mathrm{CDCl}_{3}\right) \delta 140.7$, $139.5,135.3,134.3,132.9(\mathrm{q}, \mathrm{J}=3.2 \mathrm{~Hz}), 129.8,129.5,128.9,128.3(\mathrm{q}, \mathrm{J}=$ $\left.277.7 \mathrm{~Hz}, \mathrm{CF}_{3}\right), 127.9,127.4,127.1,127.1,43.8(\mathrm{q}, \mathrm{J}=27.7 \mathrm{~Hz}),-3.4,-3.5 .{ }^{19} \mathrm{~F}$ $\operatorname{NMR}\left(376 \mathrm{MHz} \mathrm{CDCl}_{3}\right) \delta-56.5$ (t, J $\left.=13.0 \mathrm{~Hz}, 3 \mathrm{~F}\right)$. IR (neat) $\mathrm{v} / \mathrm{cm}^{-1} 3071,3031$, 2963, 2905, 2362, 2284, 1488, 1239, 1151, 1133, 1068, 810, 764, 739, 695, 649. HRMS (EI) calculated for $\mathrm{C}_{22} \mathrm{H}_{21} \mathrm{~F}_{3} \mathrm{Si}^{+}\left(\mathrm{M}^{+}\right)$: 370.1359 , observed: 370.1363 . The asymmetric reaction was performed using general procedure $F$, with substitution of $n \mathrm{Bu}_{3} \mathrm{SnH}$ for $\mathrm{PhMe} 2 \mathrm{SiH}(15.3 \mu \mathrm{L}, 1.0 \mathrm{eq}, 0.1 \mathrm{mmol})$. Purified by silica gel column chromatography $\left(5-15 \% \mathrm{CH}_{2} \mathrm{Cl}_{2}\right.$ in pentane). The product (+)(2m) was obtained in $65 \%$ and $99 \%$ ee, HPLC conditions; Chiralcel OJ-H column, hexane $/ \mathrm{PrOH}=99: 1$, flow rate $=1.0 \mathrm{~mL} / \mathrm{min}, 25^{\circ} \mathrm{C}$, wavelength $=250$ $\mathrm{nm}, \mathrm{tR}=15.06 \mathrm{~min}$ for major isomer, $\mathrm{tR}=20.53 \mathrm{~min}$ for minor isomer: $[\alpha]^{\mathrm{D}_{25}}$ $=+75.8$ (c 0.85, MeOH).

\section{(1-([1,1'-biphenyl]-4-yl)-2,2,2-trifluoroethyl)tributylgermane (2n)}

Prepared following general procedure $\mathrm{D}$, with substitution of $n \mathrm{Bu} \mathrm{u}_{3} \mathrm{SnH}$ for $n \mathrm{Bu}_{3} \mathrm{GeH}(25.8 \mu \mathrm{L}, 1.0 \mathrm{eq}, 0.1 \mathrm{mmol})$. Purified by silica gel column chromatography (pentane) and obtained as a colourless oil in $58 \%$ yield ( 28 $\mathrm{mg}, 0.06 \mathrm{mmol}) .{ }^{1} \mathrm{H}$ NMR $\left(400 \mathrm{MHz}, \mathrm{CDCl}_{3}\right): \delta$ 7.61-7.58 (m, 2H), 7.56-7.53 (m, $2 \mathrm{H}), 7.47-7.42(\mathrm{~m}, 2 \mathrm{H}), 7.37-7.33(\mathrm{~m}, 1 \mathrm{H}), 7.25(\mathrm{~d}, \mathrm{~J}=7.7 \mathrm{~Hz}, 2 \mathrm{H}), 3.15(\mathrm{q}, 3 \mathrm{JHF}$ $=13.2 \mathrm{~Hz}, 1 \mathrm{H}), 1.34-1.24(\mathrm{~m}, 12 \mathrm{H}), 0.92-0.83(\mathrm{~m}, 15 \mathrm{H}) .{ }^{13} \mathrm{C} N M R(101 \mathrm{MHz}$, $\mathrm{CDCl}_{3}$ ): $\delta 140.8,139.3,134.3,129.0,128.9\left(\mathrm{q},{ }^{1} \mathrm{~J}_{\mathrm{CF}}=276.8 \mathrm{~Hz}\right), 128.9,127.4$, $127.3,127.1,41.0\left(q,{ }^{2} \mathrm{~J}_{\mathrm{CF}}=28.8 \mathrm{~Hz}\right), 27.0,26.6,13.8,12.7 .{ }^{19} \mathrm{~F} \mathrm{NMR}(377 \mathrm{MHz}$, $\mathrm{CDCl}_{3}$ ): $\delta-57.1\left(\mathrm{~d},{ }^{3} \mathrm{~J}_{\mathrm{FH}}=13.2 \mathrm{~Hz}, 3 \mathrm{~F}\right) . \mathrm{IR}$ (neat) $\mathrm{U} / \mathrm{cm}^{-1}: 3032,2956,2927,2871$ $2857,1910,1600,1520,1488,1464,1416,1378,1344,1306,1281,1262$, $1242,1205,1141,1131,1109,1063,1008,964,912,884,855,826,765,743$, $729,695,681,654,616$. HRMS $(\mathrm{Cl})$ calculated for $\mathrm{C}_{26} \mathrm{H}_{41} \mathrm{~F}_{3} \mathrm{GeN}^{+}\left(\left[\mathrm{M}+\mathrm{NH}_{4}\right]^{+}\right)$: 498.2397, observed: 498.2435 . The asymmetric reaction was performed using general procedure $\mathrm{F}$, with substitution of $n \mathrm{Bu}_{3} \mathrm{SnH}$ for $n \mathrm{Bu}_{3} \mathrm{GeH}(25.8 \mu \mathrm{L}, 1.0$ eq, $0.1 \mathrm{mmol})$. The product $(+)-(2 \mathrm{n})$ was obtained in $99 \%$ yield with this procedure. $99 \%$ ee, HPLC conditions; Chiralpak IC column, hexane $=100 \%$, flow rate $=1.0 \mathrm{~mL} / \mathrm{min}, 25{ }^{\circ} \mathrm{C}$, wavelength $=254 \mathrm{~nm}, \mathrm{tR}=6.15 \mathrm{~min}$ for major isomer, $\mathrm{tR}=6.97 \mathrm{~min}$ for minor isomer: $[\alpha]^{\mathrm{D}_{25}}=+54.3$ (c $3.46, \mathrm{MeOH}$ ).

\section{Acknowledgments}

Financial support was provided by the EPSRC (S.H.), a CASE Studentship with AstraZeneca (S.H.), the Swiss National Foundation (J.V.), and the EPSRC Centre for Doctoral Training in Synthesis for Biology and Medicine (EP/L015838/1) (D. M. H. A. and R. S.).

\section{References and notes}

P. Yates, J. Am. Chem. Soc., 1952, 74, 5376-5381.

H. M. L. Davies and S. J. Hedley, Chem. Soc. Rev., 2007, 36, 1109. D. Gillingham and N. Fei, Chem. Soc. Rev., 2013, 42, 4918. A. Ford, H. Miel, A. Ring, C. N. Slattery, A. R. Maguire and M. A. McKervey, Chem. Rev., 2015, 115, 9981-10080.

M. P. Doyle, R. Duffy, M. Ratnikov and L. Zhou, Chem. Rev., 2010, 110, 704-724.

S. Jia, D. Xing, D. Zhang and W. Hu, Angew. Chemie Int. Ed., 2014 53, 13098-13101.

V. Bagheri, M. P. Doyle, J. Taunton and E. E. Claxton, J. Org. Chem., 1988, 53, 6158-6160.

R. Paulissen, E. Hayez, A. J. Hubert and P. Teyssie, Tetrahedron Lett., 1974, 15, 607-608.

H. E. Bartrum, D. C. Blakemore, C. J. Moody and C. J. Hayes, Tetrahedron, 2013, 69, 2276-2282.

X. Zhang and Z. Sui, Tetrahedron Lett., 2006, 47, 5953-5955. Q.-Q. Cheng, S.-F. Zhu, Y.-Z. Zhang, X.-L. Xie and Q.-L. Zhou, J. Am. Chem. Soc., 2013, 135, 14094-14097. 
C. N. Slattery, A. Ford and A. R. Maguire, Tetrahedron, 2010, 66, 6681-6705.

S.-F. Zhu and Q.-L. Zhou, Acc. Chem. Res., 2012, 45, 1365-1377. A. Padwa and D. Eastman, J. Org. Chem., 1969, 34, 2728-2732.

M. P. Doyle, J. Taunton, S. Oon, M. T. H. Liu, N. Soundararajan, M. S. Platz and J. E. Jackson, Tetrahedron Lett., 1988, 29, 5863-5866. J. E. Jackson, N. Soundararajan, M. S. Platz, M. P. Doyle and M. T. H. Liu, Tetrahedron Lett., 1989, 30, 1335-1338.

$\mathrm{O}$. Andrey, Y. Landais, D. Planchenault and V. Weber, Tetrahedron, 1995, 51, 12083-12096.

Z. Tan, Z. Qu, B. Chen and J. Wang, Tetrahedron, 2000, 56, 74577461.

D. Qiu, S. Wang, H. Meng, S. Tang, Y. Zhang and J. Wang, J. Org. Chem., 2017, 82, 624-632.

Z. Liu, Q. Li, Y. Yang and X. Bi, Chem. Commun., 2017, 53, 25032506.

S. Hyde, J. Veliks, B. Liégault, D. Grassi, M. Taillefer and V. Gouverneur, Angew. Chemie Int. Ed., 2016, 55, 3785-3789. J. R. Denton, D. Sukumaran and H. M. L. Davies, Org. Lett., 2007, 9, 2625-2628.

E. Emer, J. Twilton, M. Tredwell, S. Calderwood, T. L. Collier, B. Liégault, M. Taillefer and V. Gouverneur, Org. Lett., 2014, 16, 6004-6007. Burant, S. Dapprich, J. M. Millam, A. D. Daniels, K. N. Kudin, M. C. Strain, O. Farkas, J. Tomasi, V. Barone, M. Cossi, R. Cammi, B. Mennucci, C. Pomelli, C. Adamo, S. Clifford, J. Ochterski, G. A. Petersson, P. Y. Ayala, Q. Cui, K. Morokuma, N. Roga, P. Salvador, J. J. Dannenberg, D. K. Malick, A. D. Rabuck, K. Raghavachari, J. B. Foresman, J. Cioslowski, J. V. Ortiz, A. G. Baboul, B. B. Stefanov, G. Liu, A. Liashenko, P. Piskorz, I.Komaromi, R. Gomperts, R. L. Martin,

D. J. Fox, T. Keith, M. A. Al-Laham, C. Y. Penng, A. Nanayakkara, M. Challacombe, P. M. W. Gill, B. Johnson, W. Chen, M. W. Wong, J. L. Andres, C. Gonzalez, M. Head-Gordon, E. S. Replogle and J. A. Pople, 2003. Comput. Theor. Chem., 2014, 1050, 7-14.

S. R. Whittleton, R. J. Boyd and T. B. Grindley, Can. J. Chem., 2009, 87, 974-983.

L. A. Curtiss, P. C. Redfern and K. Raghavachari, J. Chem. Phys., 2007, 126, 084108.

M. Ueda and H. Ito, J. Polym. Sci. Part A Polym. Chem., 1988, 26, 89-98.

H. Tsutsui, M. Matsuura, K. Makino, S. Nakamura, M. Nakajima, S. Kitagaki and S. Hashimoto, Isr. J. Chem., 2001, 41, 283-296.

K. Minami, H. Saito, H. Tsutsui, H. Nambu, M. Anada and S. Hashimoto, Adv. Synth. Catal., 2005, 347, 1483-1487.

M. Brambilla and M. Tredwell, Angew. Chemie Int. Ed., 2017, 56, 11981-11985.

J. Wiedemann, T. Heiner, G. Mloston, G. K. S. Prakash and G. A. Olah, 1998, 820-821.

X. Wang, Y. Xu, Y. Deng, Y. Zhou, J. Feng, G. Ji, Y. Zhang and J. Wang, Chem. - A Eur. J., 2014, 20, 961-965.

J. R. Fulton, V. K. Aggarwal and J. De Vicente, European J. Org. Chem., 2005, 1479-1492.

G. Shi and Y. Xu, J. Fluor. Chem., 1990, 46, 173-178. J. Wang, J. Kubicki, T. L. Gustafson and M. S. Platz, J. Am. Chem. Soc., 2008, 130, 2304-2313.

B. Raimer and T. Lindel, Chem. - A Eur. J., 2013, 19, 6551-6555. For most stannanes, the molecular ion peak was not detected; the relevant fragment masses are therefore reported. Compound unstable under various ionisation techniques and HRMS could therefore not be obtained. 